\title{
Boosting Photoelectric Conductivity in Porphyrin-Based MOFs Incorporating $\mathbf{C}_{60}$
}

\section{Saied Md Pratik, Laura Gagliardi*, Christopher J. Cramer*}

Department of Chemistry, Minnesota Supercomputing Institute, and Chemical Theory Center, University of Minnesota, Minneapolis, Minnesota 55455, United States

Corresponding Authors

*E-mail: gagliard@umn.edu and cramer@umn.edu.

\section{Contents:}

Section S1. Structural and electronic properties from periodic calculations.

Section S2. Cluster models and Excited states calculations.

Section S3. Anisotropic hopping pathways for hole and electron migration.

Section S4. Full references of Gaussian 16 and ADF. 
Section S1. Structural and electronic properties from periodic calculations.

TableS1. Experimental and computed lattice parameters for DA-MOF, F-MOF and their derivatives before and after $\mathrm{C}_{60}$ installation. Computed total electronic energies $\left(\mathrm{E}_{\text {tot }}\right)$ for the corresponding MOFs are reported in $\mathrm{eV}$.

\begin{tabular}{|c|c|c|c|c|c|c|c|}
\hline MOF & a $(\AA)$ & b $(\AA)$ & c $(\AA)$ & $\alpha\left({ }^{\circ}\right)$ & $\beta\left(^{\circ}\right)$ & $\gamma\left({ }^{\circ}\right)$ & $\mathrm{E}_{\text {tot }}(\mathrm{eV})$ \\
\hline \multicolumn{7}{|c|}{ Experimental } & \\
\hline DA-MOF & 11.47 & 15.59 & 27.17 & 92.2 & 93.4 & 90.0 & \\
\hline F-MOF & 11.57 & 15.45 & 21.19 & 76.8 & 79.0 & 89.6 & \\
\hline \multicolumn{7}{|c|}{ Calculated } & \\
\hline DA-MOF & 11.44 & 15.70 & 27.32 & 92.6 & 95.1 & 90.0 & -1025.535529 \\
\hline F-MOF & 11.58 & 15.46 & 21.09 & 76.6 & 79.1 & 89.7 & -995.6896301 \\
\hline \multicolumn{7}{|c|}{ Functionalized parent MOFs } & \\
\hline H_DA-MOF & 11.48 & 15.72 & 27.38 & 92.6 & 95.3 & 90.1 & -883.9122123 \\
\hline $\mathrm{NH}_{2}$ DA-MOF & 11.48 & 15.72 & 27.38 & 92.6 & 95.3 & 90.1 & -908.9274178 \\
\hline H_F-MOF & 11.63 & 15.48 & 21.10 & 76.6 & 79.2 & 89.7 & -849.9609226 \\
\hline $\mathrm{NH}_{2}$ F- MOF & 11.44 & 15.65 & 20.78 & 76.1 & 80.7 & 89.8 & -874.9060694 \\
\hline \multicolumn{7}{|c|}{$\mathrm{C}_{60}$ incorporated parent $\mathrm{MOF}$} & \\
\hline DA-MOF & 11.43 & 15.74 & 27.48 & 91.9 & 93.3 & 90.0 & -1585.402782 \\
\hline F-MOF & 12.45 & 15.03 & 22.32 & 76.4 & 72.31 & 89.41 & -1555.653848 \\
\hline \multicolumn{7}{|c|}{ C $60_{\text {incorporated H_DA-MOF }}$} & \\
\hline Type-I & 11.41 & 15.77 & 27.50 & 92.0 & 93.8 & 89.9 & -1443.459608 \\
\hline Type-II & 12.69 & 14.83 & 27.30 & 92.3 & 95.1 & 89.8 & -1443.706503 \\
\hline Type-III & 11.49 & 15.79 & 27.32 & 91.8 & 93.9 & 90.0 & -1443.441689 \\
\hline \multicolumn{7}{|c|}{$\mathrm{C}_{60}$ incorporated $\mathrm{NH}_{2}$ DA-MOF } & \\
\hline Type-I & 11.20 & 15.88 & 27.35 & 92.1 & 93.7 & 89.9 & -1468.628354 \\
\hline Type-II & 12.64 & 14.82 & 27.25 & 92.4 & 95.6 & 89.9 & -1468.879786 \\
\hline Type-III & 11.62 & 15.75 & 27.29 & 94.0 & 95.2 & 90.7 & -1468.508145 \\
\hline \multicolumn{7}{|c|}{$\mathrm{C}_{60}$ incorporated H_F-MOF } & \\
\hline Type-A & 12.51 & 14.87 & 22.57 & 76.3 & 71.3 & 89.5 & -1409.970403 \\
\hline
\end{tabular}




\begin{tabular}{|c|c|c|c|c|c|c|c|}
\hline Type-B & 11.63 & 15.57 & 21.12 & 76.9 & 79.4 & 90.0 & -1409.713804 \\
\hline \multicolumn{7}{|c|}{ C $_{\mathbf{6 0}}$ incorporated NH__F-MOF $^{\text {_F }}$} \\
\hline Type-A & 12.48 & 14.91 & 22.49 & 76.5 & 71.6 & 89.4 & -1434.991321 \\
\hline Type-B & 11.53 & 15.60 & 20.94 & 77.0 & 79.9 & 90.0 & -1434.904885 \\
\hline
\end{tabular}
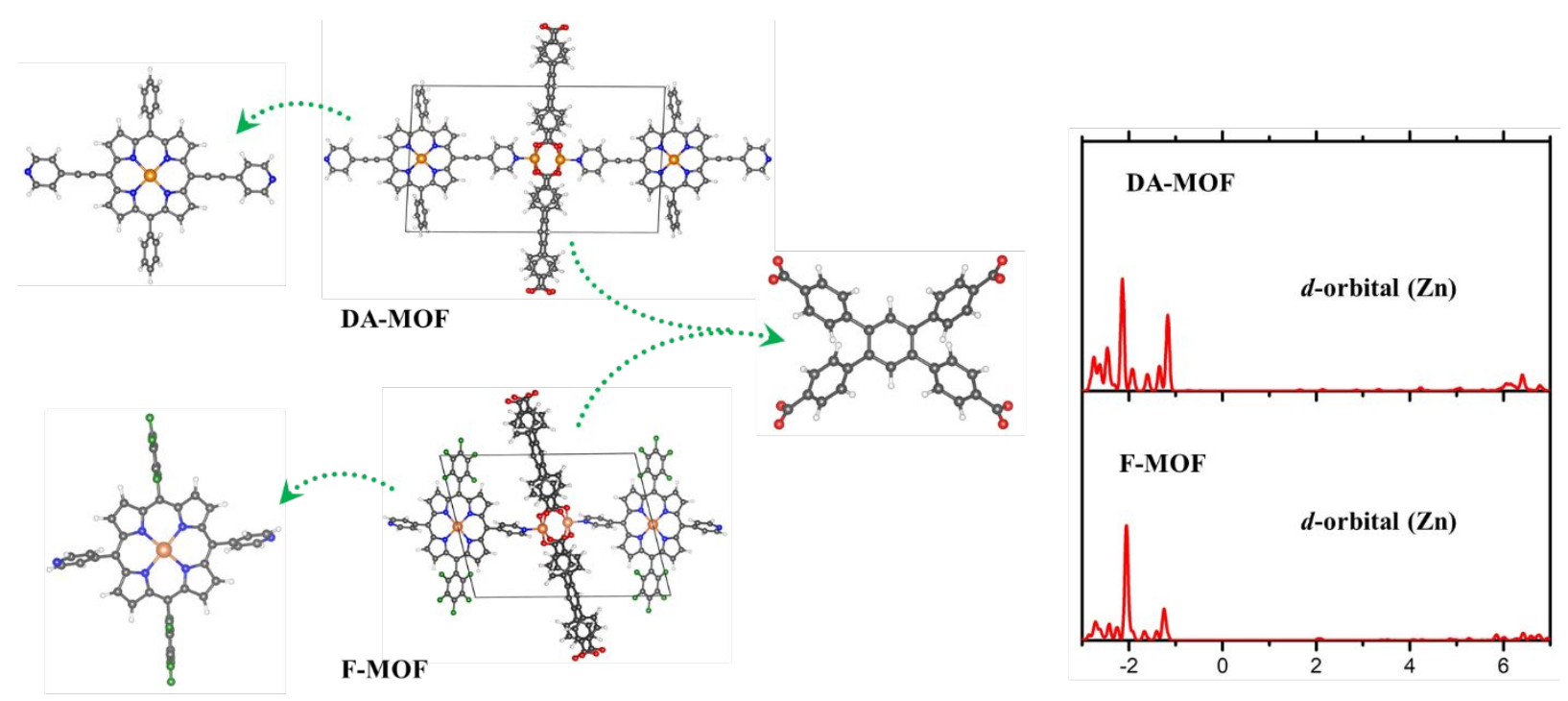

(a)

(b)

Figure S1. (a) Structure of DA- and F-MOF along with the linkers. [ $\mathrm{Zn}_{2}(\mathrm{TCPB})(\mathrm{DA}-\mathrm{ZnP})$ (DA$\mathrm{MOF}, \quad \mathrm{DA}-\mathrm{ZnP}=$ [5,15-bis[(4-pyridyl)ethynyl]-10,20-diphenylporphinato]zinc(II)) and $\mathrm{Zn}_{2}$ (TCPB)(F-ZnP) (F-MOF, $\mathrm{H}_{4} \mathrm{TCPB}=1,2,4,5$-tetrakis(4-carboxyphenyl)benzene, $\mathrm{F}-\mathrm{ZnP}=$ [5,15-di(4-pyridyl)-10,20-bis(pentafluorophenyl)porphinato]-zinc(II))]. Atomic colors are $\mathrm{H}$ (white), C (gray), N (blue), O (red), F (blue-green), and Zn (orange). (b) computed PDOS arising from the d-orbital of $\mathrm{Zn}$ for both DA-MOF and F-MOF. 


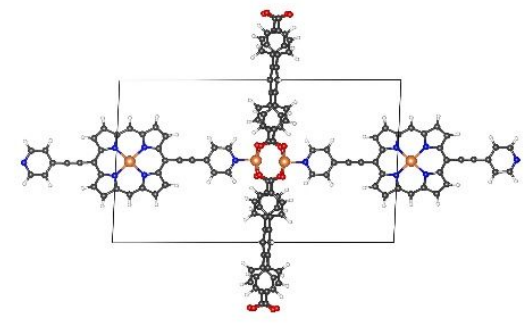

(a)

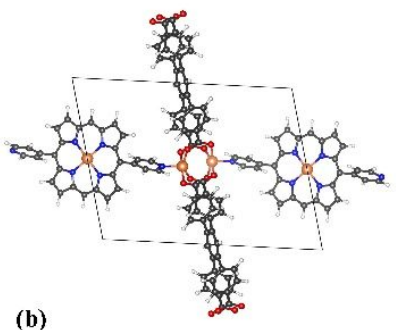

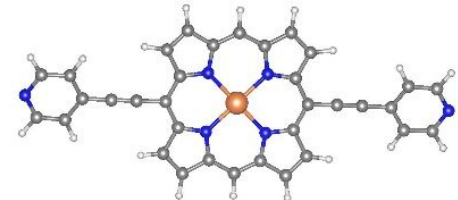

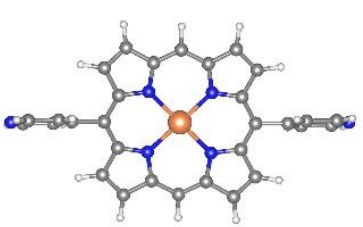

Figure S2. Optimized structures of (a) H_DA-MOF and (b) H_F-MOF are shown in left panel. In right panel associated porphyrin-based linkers are shown.

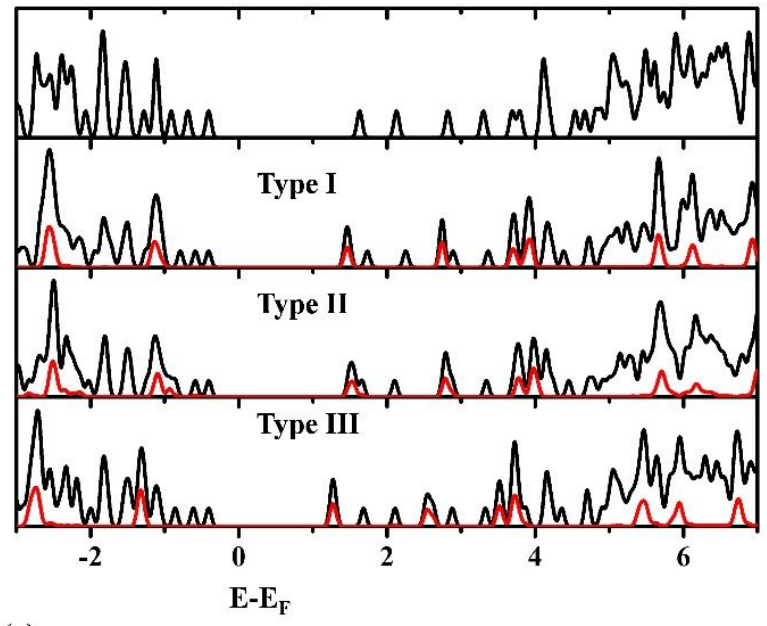

(a)

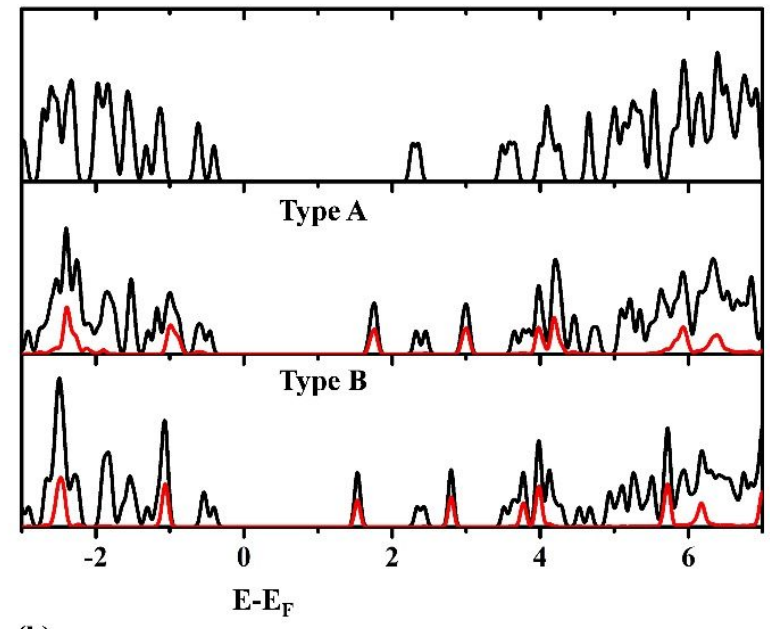

(b)

Figure S3. Calculated TDOS for (a) H_DA and (b) H_F-MOF are shown in upper panel. In other panels TDOS (black line) and PDOS of $\mathrm{C}_{60}$ (red line) for different interfaces are shown. The energy is scaled with respect to the Fermi energy. 

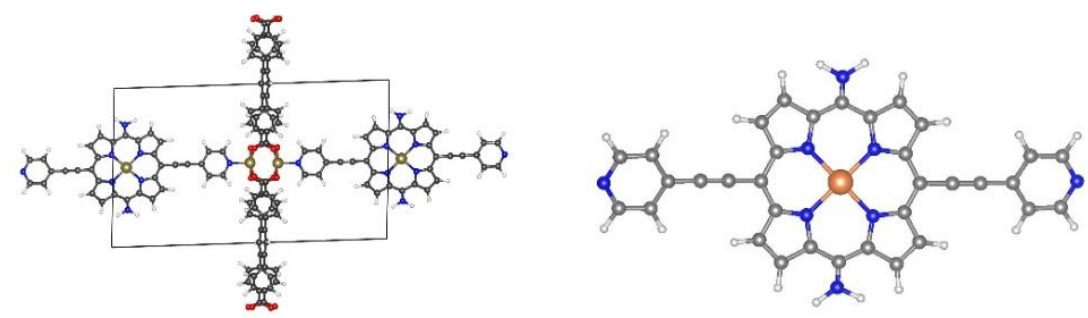

(a)

(b)
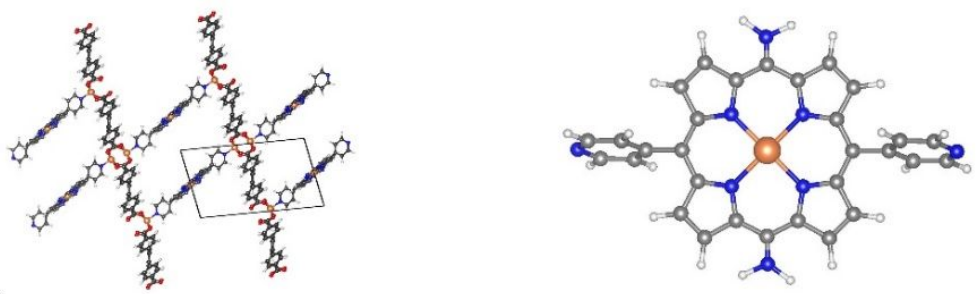

Figure S4. Optimized structures of (a) $\mathrm{NH}_{2}$ DA-MOF and (b) $\mathrm{NH}_{2}$ F-MOF are shown in left panel. In right panel, corresponding $-\mathrm{NH}_{2}$ functionalized porphyrin-based linkers are shown.
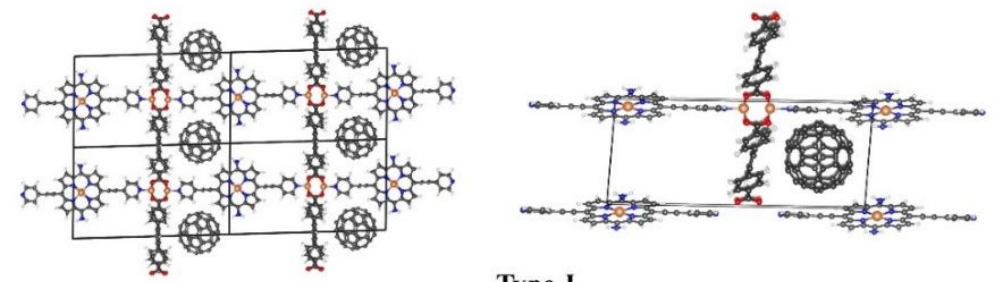

Type-I
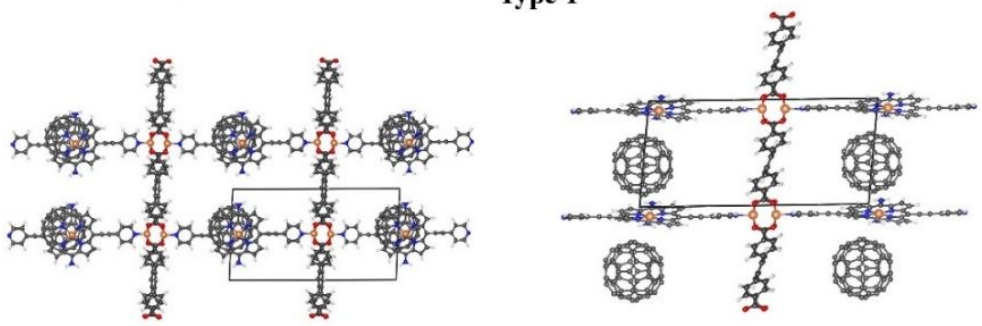

Type-II
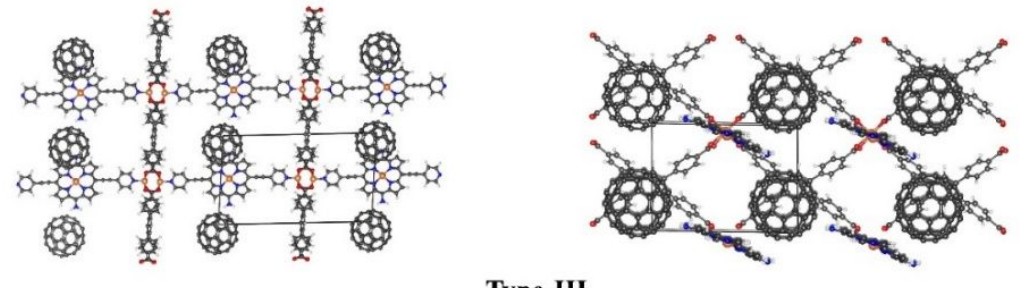

Figure S5. Optimized structures of $\mathrm{NH}_{2}$ DA-MOF for Type-I, Type-II and Type-III interfaces (side and top view). 

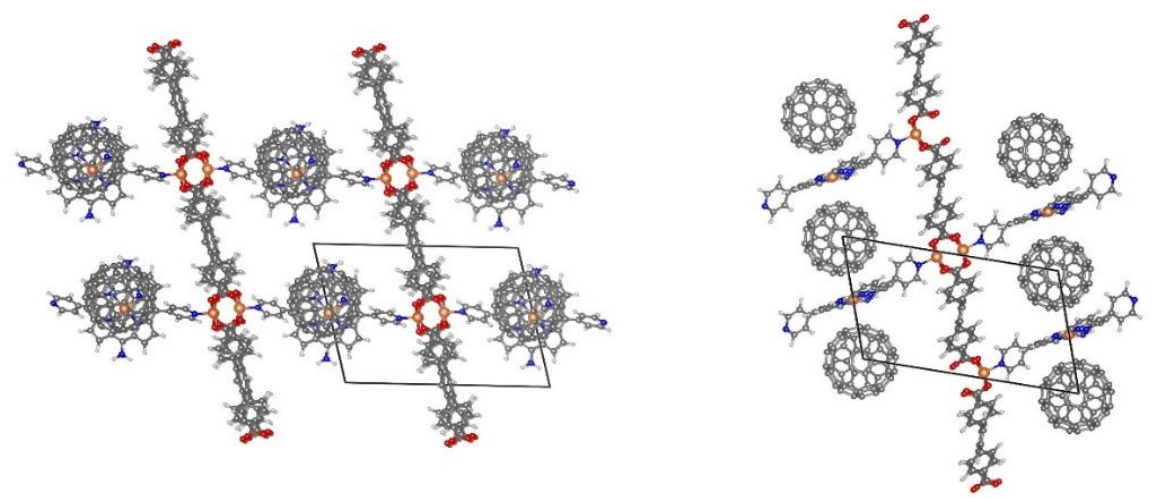

Type-A
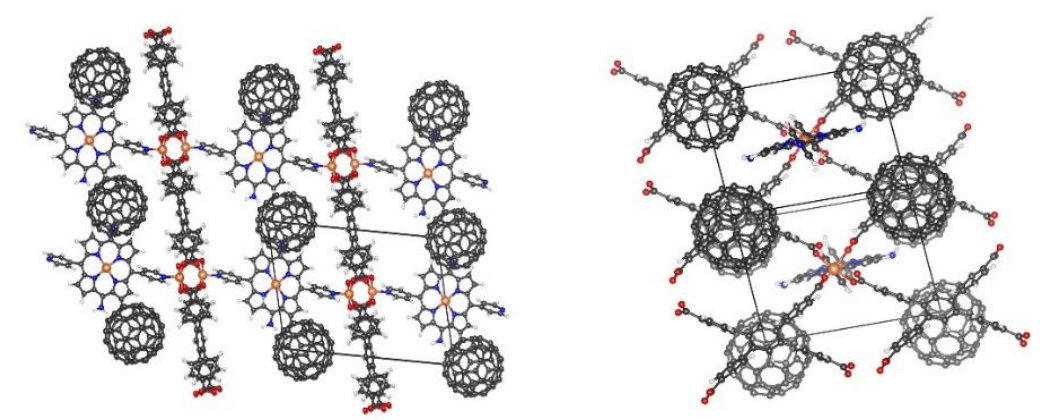

Type-B

Figure S6. Optimized structures of $\mathrm{NH}_{2}$ F-MOF for Type-A and Type-B interfaces (side and top view). 


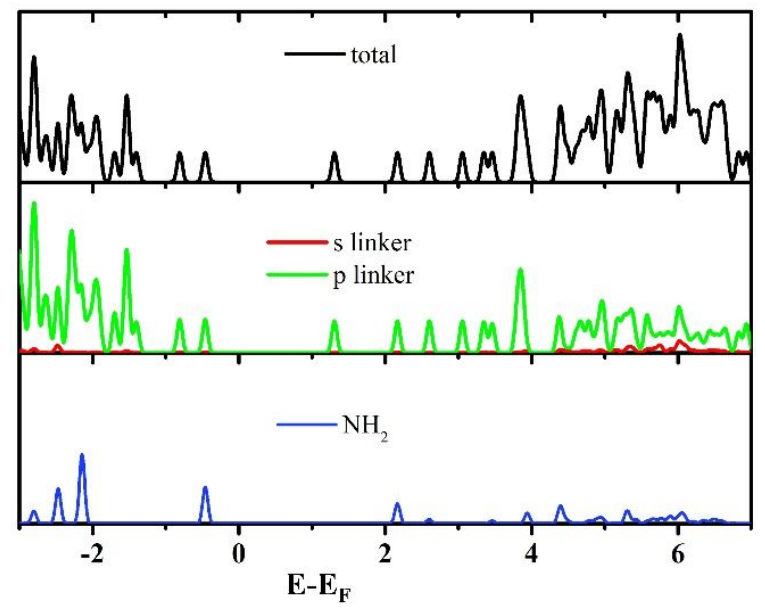

(a)

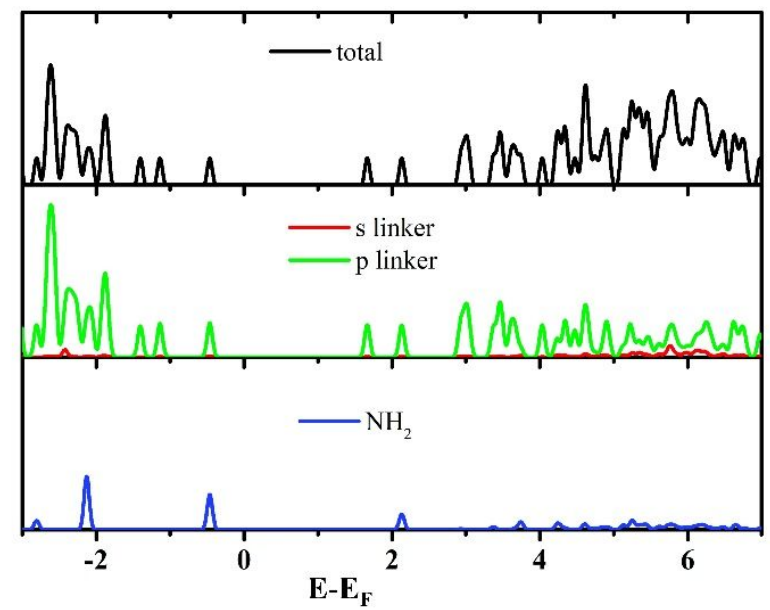

(b)

Figure S7. Calculated TDOS for (a) H_DA and (b) H_F-MOF. Contribution from $s$ - and $p$ orbitals are shown in middle panel. The electronic states arising due to $-\mathrm{NH}_{2}$ are shown in lower panel. The energy is scaled with respect to the Fermi energy.

\section{Section S2. Cluster models and Excited states calculations.}

Cluster models and their fragments:

Visual inspection clearly demonstrates (Figure S8-S14) that each $\mathrm{C}_{60}$ is in the close proximity of four porphyrin units within the unit cell. Therefore, in principle we should consider all four porphyrins along with the $\mathrm{C}_{60}$ in order to design the cluster models. However, to reduce the computation cost, we have decomposed these clusters into different fragments based on the center to center distances between porphyrin-based linkers and $\mathrm{C}_{60}$ as shown below. The cluster models for $\mathrm{C}_{60}$ incorporated DA-MOF, F-MOF, H-DA-MOF and H-F-MOF are shown below. For $\mathrm{C}_{60}$ incorporated $\mathrm{NH}_{2}$ DA-MOF and $\mathrm{NH}_{2}$ F-MOF we have considered similar fragments as we have considered for H-DA-MOF and H-F-MOF. 


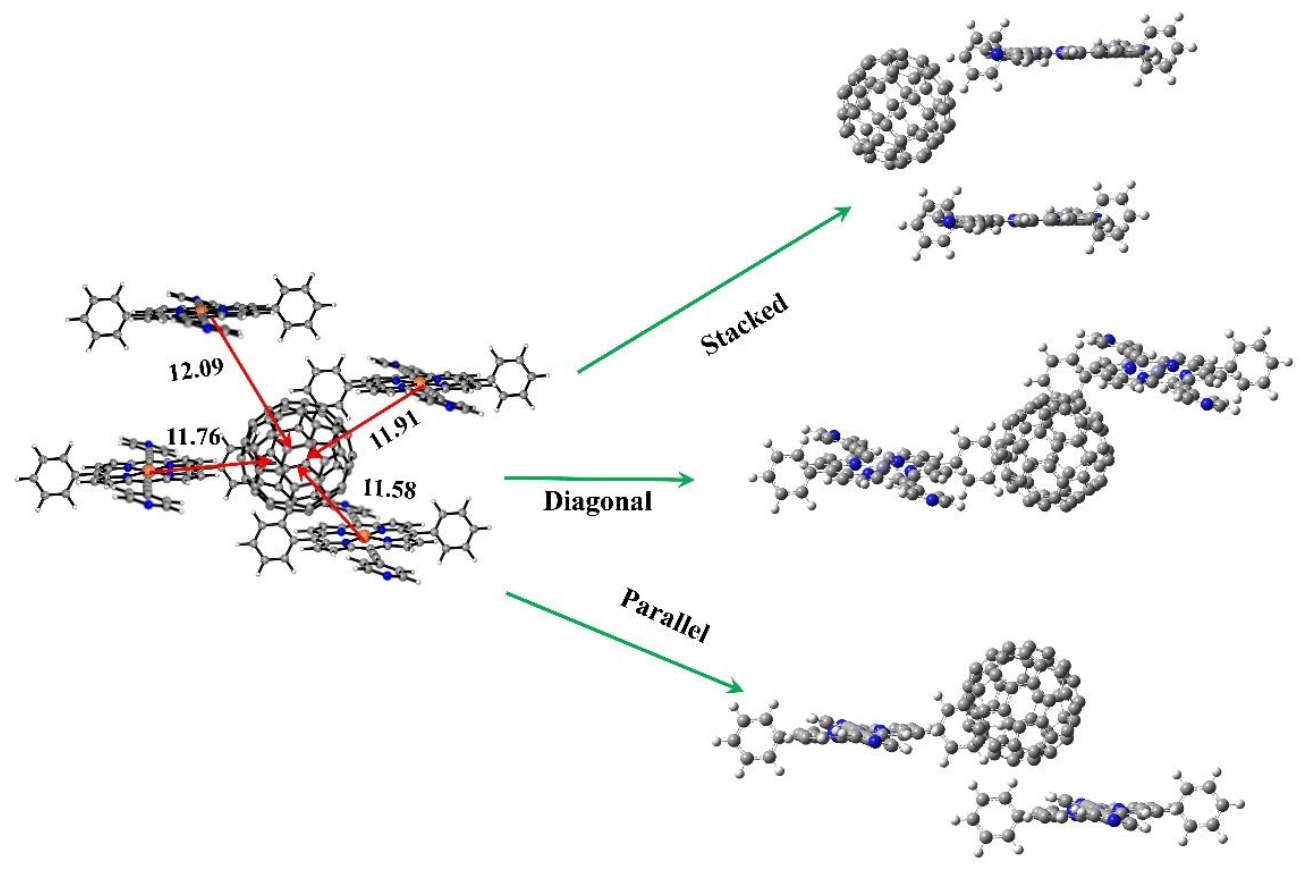

Figure S8. Cluster model of $\mathrm{C}_{60}$ incorporated DA-MOF and different fragments for TD-DFT calculations. Center to center distances between porphyrin and $\mathrm{C}_{60}$ are shown in $\AA$.
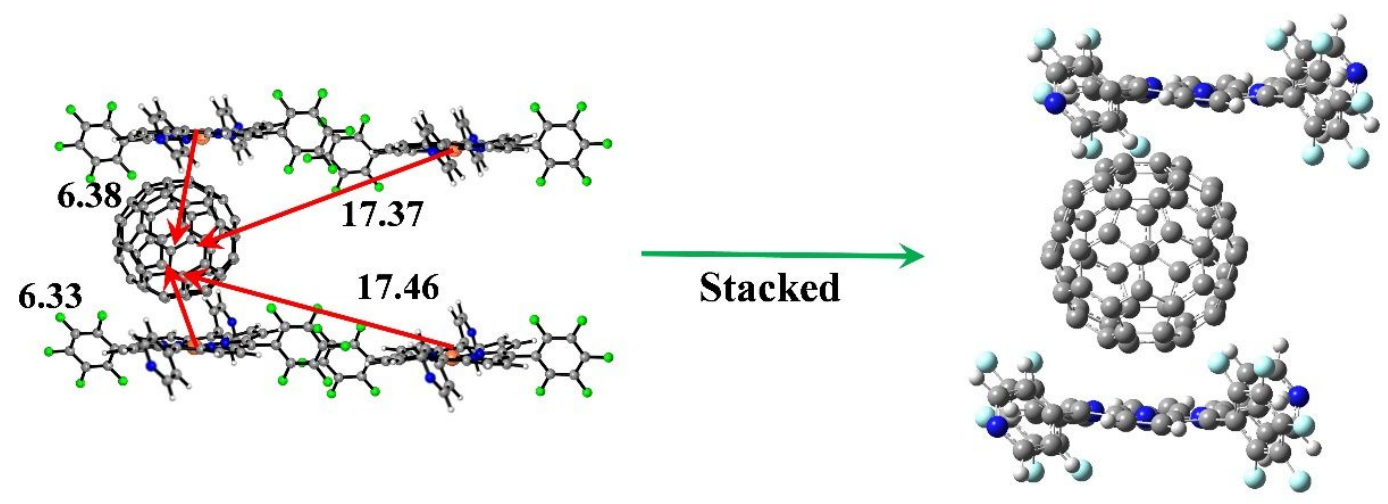

Figure S9. Cluster model of $\mathrm{C}_{60}$ incorporated F-MOF and stacked fragment for TD-DFT calculation. Center to center distances between porphyrin and $\mathrm{C}_{60}$ are shown in $\AA$. 

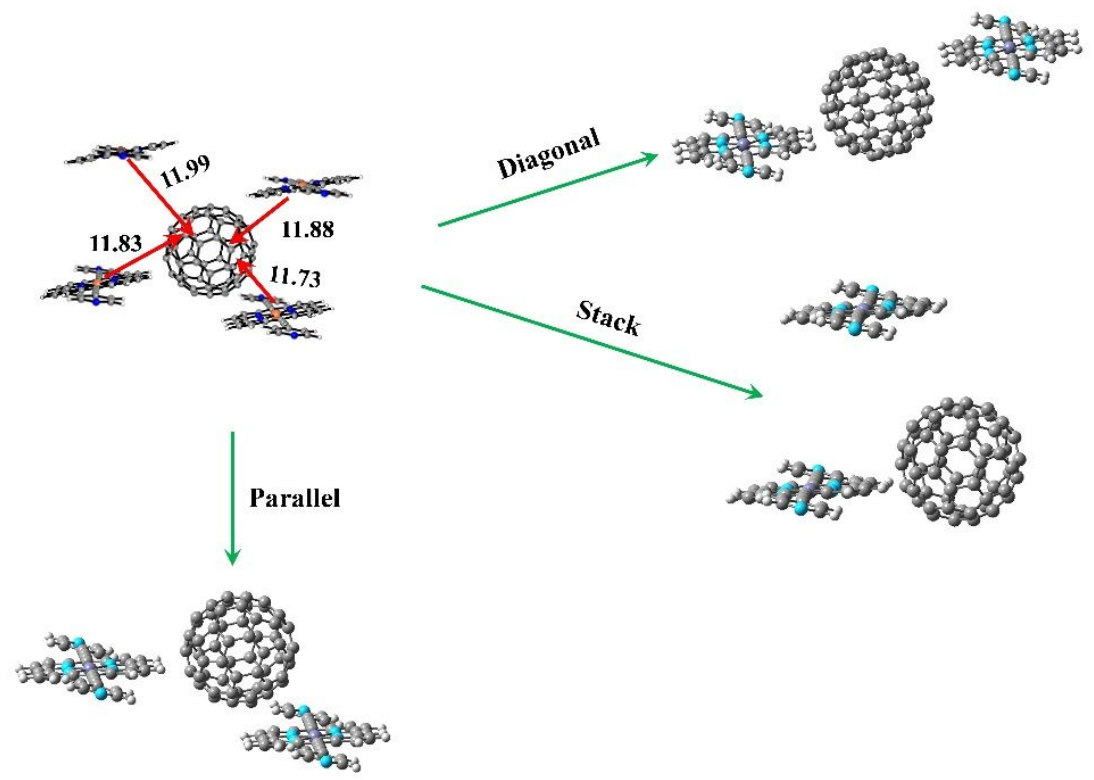

Figure S10. Cluster model of $\mathrm{C}_{60}$ incorporated H_DA-MOF in Type-I interface and different fragments for TD-DFT calculations. Center to center distances between porphyrin and $\mathrm{C}_{60}$ are shown in $\AA$.
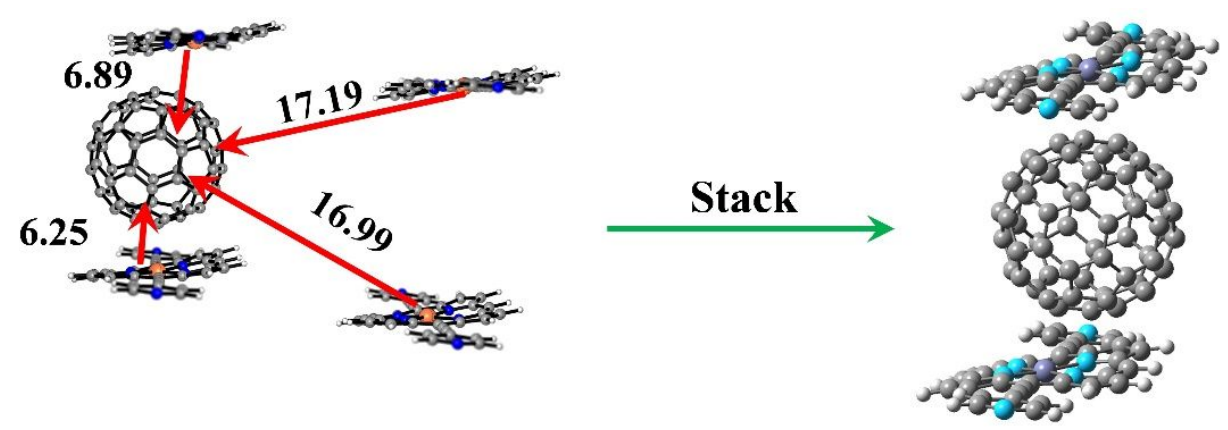

Figure S11. Cluster model of $\mathrm{C}_{60}$ incorporated H_DA-MOF in Type-II interface and stacked fragment for TD-DFT calculation. Center to center distances between porphyrin and $\mathrm{C}_{60}$ are shown in $\AA$. 


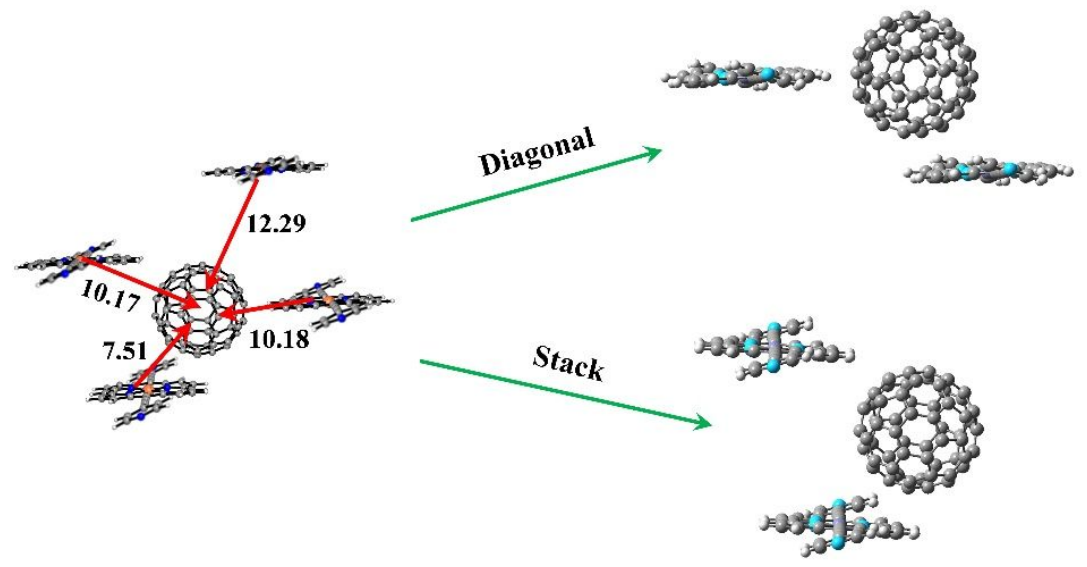

Figure S12. Cluster model of $\mathrm{C}_{60}$ incorporated H_DA-MOF in Type-III interface and different fragments for TD-DFT calculations. Center to center distances between porphyrin and $\mathrm{C}_{60}$ are shown in $\AA$.
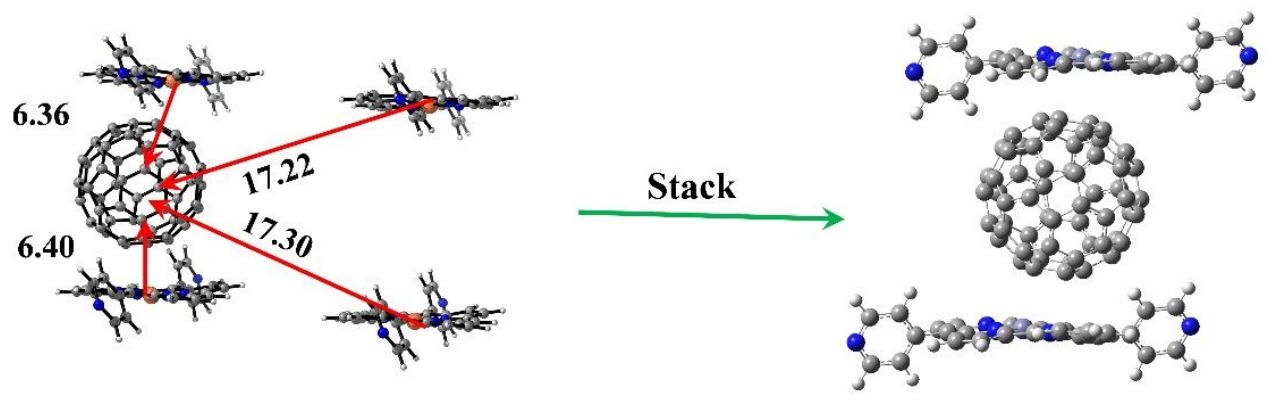

Figure S13. Cluster model of $\mathrm{C}_{60}$ incorporated H_F-MOF in Type-A interface and stacked fragment for TD-DFT calculation. Center to center distances between porphyrin and $\mathrm{C}_{60}$ are shown in $\AA$. 


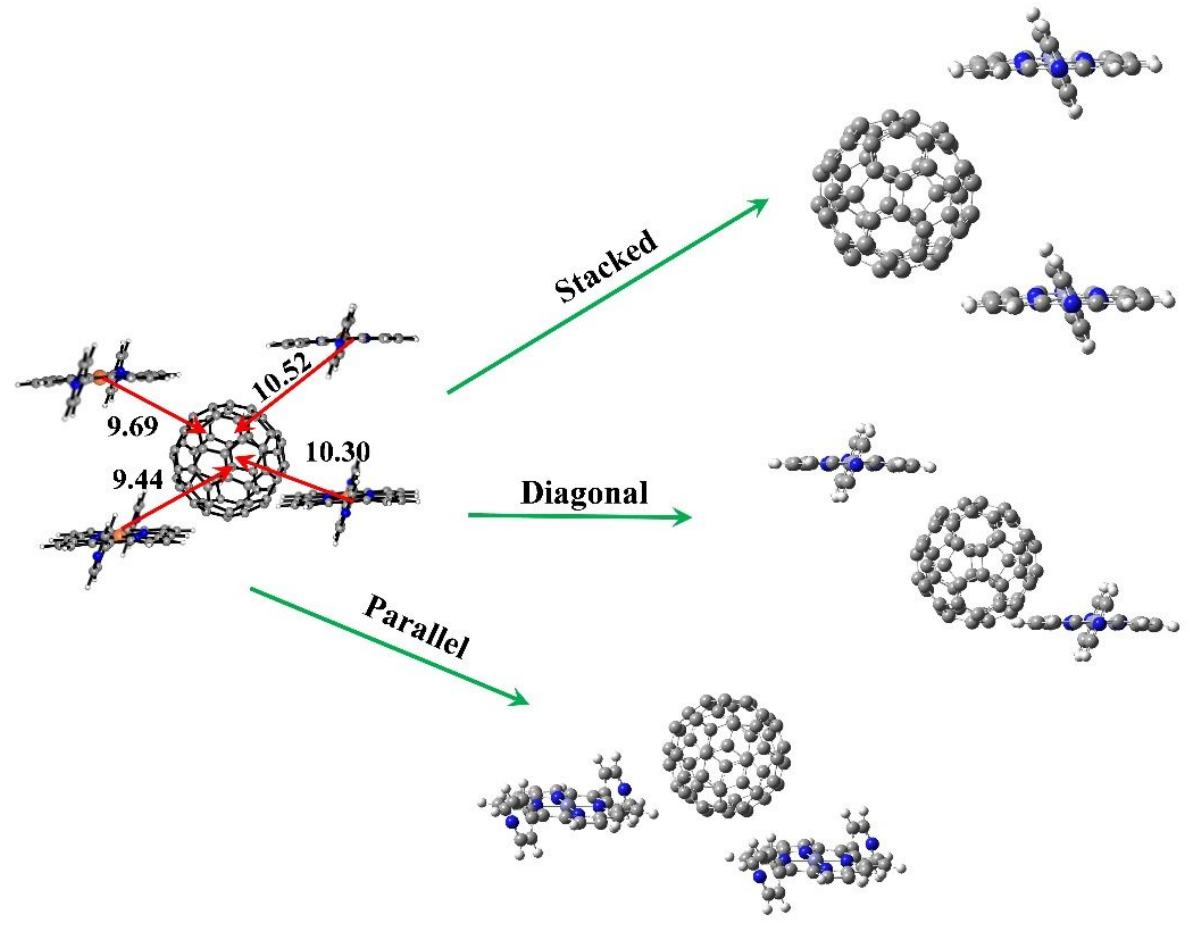

Figure S14. Cluster model of $\mathrm{C}_{60}$ incorporated H_F-MOF in Type-B interface and different fragments for TD-DFT calculations. Center to center distances between porphyrin and $\mathrm{C}_{60}$ are shown in $\AA$. 
Excited states calculations:

S2.1. Results for $\mathrm{C}_{60}$ incorporated DA-MOF:

\begin{tabular}{|c|c|c|c|c|c|}
\hline \multirow[t]{2}{*}{ Stacked } & 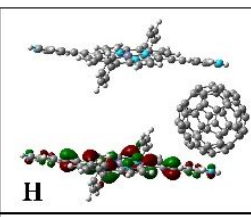 & 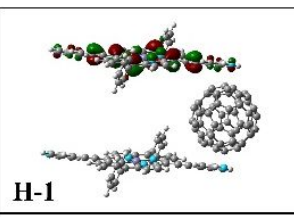 & H-2 & 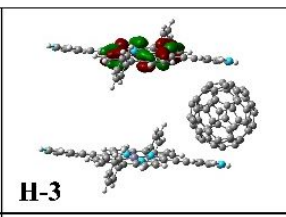 & 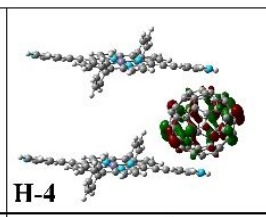 \\
\hline & L & L+1 & 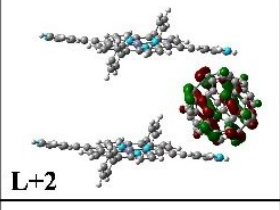 & 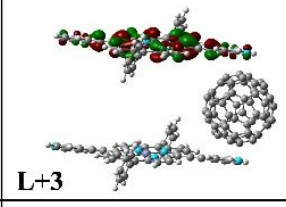 & 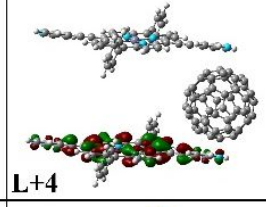 \\
\hline \multirow[t]{2}{*}{ Parallel } & H & H-1 & H-2 & 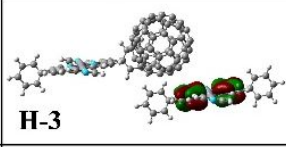 & $\begin{array}{l}\text { H-4 } \\
\text { Hons }\end{array}$ \\
\hline & $\mathbf{L}$ & $\mathrm{L}+1$ & $\mathbf{L}+2$ & $\mathbf{L}+3$ & $L+4$ \\
\hline \multirow[t]{2}{*}{ Diagonal } & H & 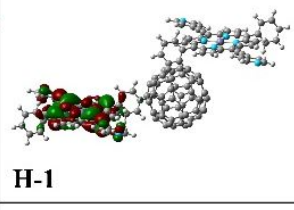 & $\begin{array}{ll} & \\
\text { H-2 }\end{array}$ & 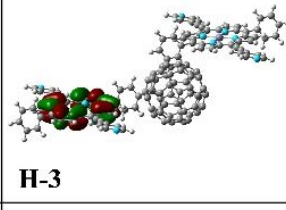 & H-4 \\
\hline & $\begin{array}{l}\text { L } \\
\text { L }\end{array}$ & 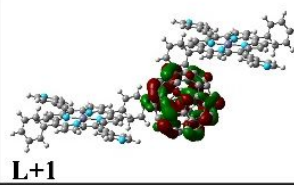 & $\begin{array}{l}\text { bis } \\
\mathrm{L}+2\end{array}$ & $\begin{array}{l}\text { ats } \\
\mathrm{L}+3\end{array}$ & $\begin{array}{ll}2 \\
L+4\end{array}$ \\
\hline
\end{tabular}

Figure S15. Frontier molecular orbitals for DA-MOF in stacked, parallel and diagonal fragments. 
Table S2. Excited States, excitation energies (eV), wavelength (nm), oscillator strengths (f) and important orbitals involved in transition for DA-MOF in their stacked, parallel and diagonal fragments.

\begin{tabular}{|c|c|c|c|c|c|}
\hline $\begin{array}{l}\text { Systems } \\
\text { with } \mathrm{C}_{60}\end{array}$ & $\begin{array}{l}\text { Excited State } \\
\text { (Ex. St.) }\end{array}$ & $\begin{array}{c}\text { Excitation energies } \\
\qquad\left(\mathrm{E}_{0-\mathrm{n},}, \mathrm{eV}\right)\end{array}$ & $\begin{array}{c}\text { Wavelength } \\
\text { (nm) }\end{array}$ & $\begin{array}{c}\text { Oscillator } \\
\text { strengths } \\
(f)\end{array}$ & $\begin{array}{c}\text { Important } \\
\text { Orbitals } \\
\text { involved }\end{array}$ \\
\hline \multirow{7}{*}{ Stacked } & 1 & 1.3930 & 890.05 & 0.0001 & $\mathrm{H} \rightarrow \mathrm{L}$ \\
\hline & 3 & 1.3982 & 886.76 & 0.0001 & $\mathrm{H}-1 \rightarrow \mathrm{L}+1$ \\
\hline & 15 & 2.0730 & 598.09 & 0.0012 & $\begin{array}{c}\mathrm{H}-3 \rightarrow \mathrm{L}+5 \\
\mathrm{H}-1 \rightarrow \mathrm{L}+3 \\
\mathrm{H} \rightarrow \mathrm{L}+4\end{array}$ \\
\hline & 23 & 2.0960 & 591.53 & 0.9570 & $\begin{array}{r}\mathrm{H}-3 \rightarrow \mathrm{L}+5 \\
\mathrm{H}-2 \rightarrow \mathrm{L}+6 \\
\mathrm{H}-1 \rightarrow \mathrm{L}+3 \\
\mathrm{H} \rightarrow \mathrm{L}+4\end{array}$ \\
\hline & 68 & 2.9489 & 420.45 & 0.0158 & $\begin{array}{c}\mathrm{H}-3 \rightarrow \mathrm{L}+3 \\
\mathrm{H}-3 \rightarrow \mathrm{L}+9 \\
\mathrm{H}-2 \rightarrow \mathrm{L}+4 \\
\mathrm{H}-1 \rightarrow \mathrm{L}+5 \\
\mathrm{H} \rightarrow \mathrm{L}+6\end{array}$ \\
\hline & 71 & 2.9850 & 415.36 & 0.1540 & $\begin{array}{l}\mathrm{H}-12 \rightarrow \mathrm{L} \\
\mathrm{H}-3 \rightarrow \mathrm{L}+3\end{array}$ \\
\hline & 72 & 2.9854 & 415.30 & 1.4881 & $\begin{array}{l}\mathrm{H}-12 \rightarrow \mathrm{L} \\
\mathrm{H}-3 \rightarrow \mathrm{L}+3 \\
\mathrm{H}-2 \rightarrow \mathrm{L}+4 \\
\mathrm{H}-1 \rightarrow \mathrm{L}+5\end{array}$ \\
\hline
\end{tabular}




\begin{tabular}{|c|c|c|c|c|c|}
\hline & & & & & $\mathrm{H} \rightarrow \mathrm{L}+6$ \\
\hline \multirow{6}{*}{ Parallel } & 1 & 1.4431 & 859.16 & 0.0004 & $\mathrm{H} \rightarrow \mathrm{L}$ \\
\hline & 3 & 1.4521 & 853.85 & 0.0003 & $\mathrm{H} \rightarrow \mathrm{L}+1$ \\
\hline & 20 & 2.0912 & 592.87 & 0.3937 & $\begin{array}{c}\mathrm{H}-6 \rightarrow \mathrm{L}+1 \\
\mathrm{H}-5 \rightarrow \mathrm{L}+1 \\
\mathrm{H}-5 \rightarrow \mathrm{L}+2 \\
\mathrm{H}-1 \rightarrow \mathrm{L}+3 \\
\mathrm{H} \rightarrow \mathrm{L}+4\end{array}$ \\
\hline & 21 & 2.0915 & 592.81 & 0.6095 & $\begin{array}{c}\mathrm{H}-6 \rightarrow \mathrm{L}+1 \\
\mathrm{H}-5 \rightarrow \mathrm{L}+2 \\
\mathrm{H}-1 \rightarrow \mathrm{L}+3 \\
\mathrm{H} \rightarrow \mathrm{L}+4\end{array}$ \\
\hline & 62 & 2.9431 & 421.27 & 2.0350 & $\begin{array}{c}\mathrm{H}-3 \rightarrow \mathrm{L}+3 \\
\mathrm{H}-2 \rightarrow \mathrm{L}+4 \\
\mathrm{H}-1 \rightarrow \mathrm{L}+5 \\
\mathrm{H} \rightarrow \mathrm{L}+6\end{array}$ \\
\hline & 82 & 3.0674 & 404.20 & 0.5303 & $\begin{array}{r}\mathrm{H}-13 \rightarrow \mathrm{L}+2 \\
\mathrm{H}-3 \rightarrow \mathrm{L}+5 \\
\mathrm{H}-2 \rightarrow \mathrm{L}+6\end{array}$ \\
\hline \multirow{4}{*}{ Diagonal } & 1 & 1.3766 & 900.63 & 0.0002 & $\mathrm{H} \rightarrow \mathrm{L}$ \\
\hline & 2 & 1.3774 & 900.11 & 0.0003 & $\mathrm{H}-1 \rightarrow \mathrm{L}$ \\
\hline & 4 & 1.3882 & 893.15 & 0.0005 & $\mathrm{H}-1 \rightarrow \mathrm{L}+1$ \\
\hline & 20 & 2.0898 & 593.29 & 0.9466 & $\begin{array}{l}\mathrm{H}-5 \rightarrow \mathrm{L}+2 \\
\mathrm{H}-3 \rightarrow \mathrm{L}+5\end{array}$ \\
\hline
\end{tabular}




\begin{tabular}{|c|c|c|c|c|}
\hline & & & & $\mathrm{H}-2 \rightarrow \mathrm{L}+6$ \\
$\mathrm{H}-1 \rightarrow \mathrm{L}+3$ \\
\end{tabular}

S2.2. Results for $\mathrm{C}_{60}$ incorporated FA-MOF:

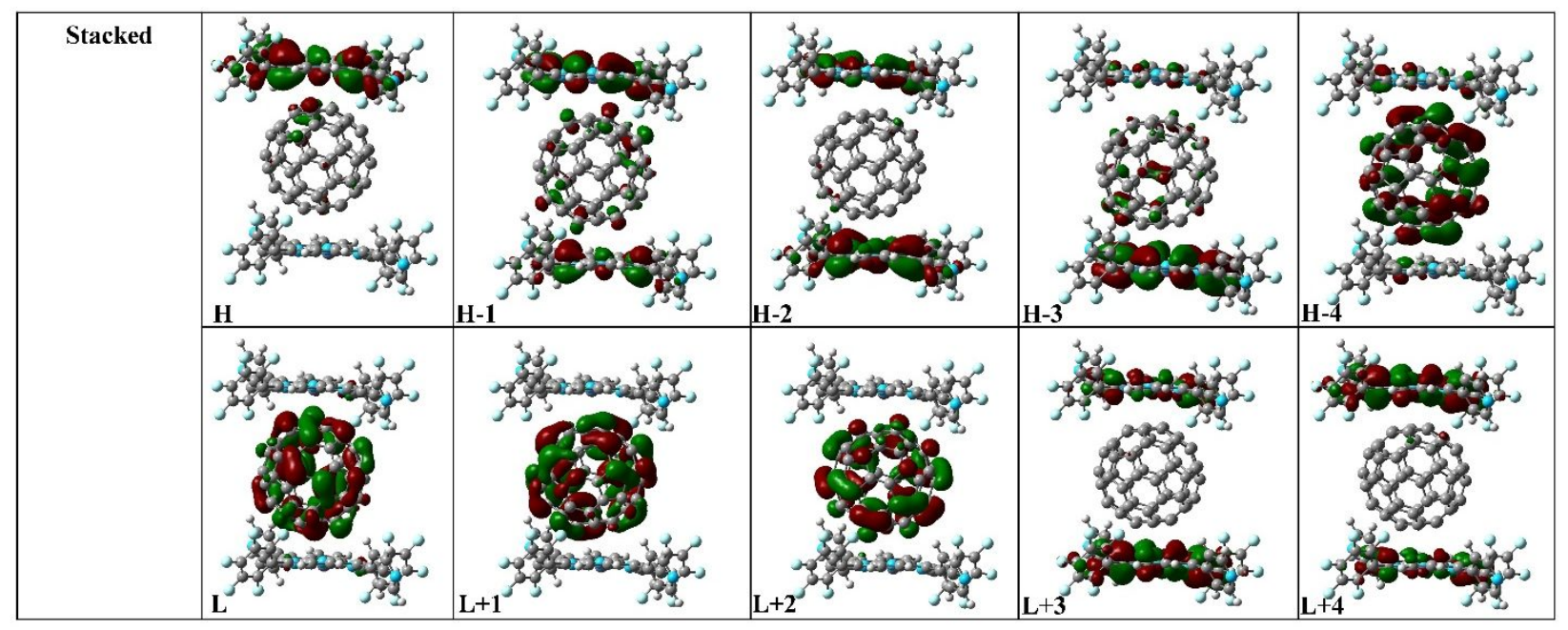

Figure S16. Frontier molecular orbitals for F-MOF in stacked fragments. 
TableS3. Excited States, excitation energies (eV), wavelength (nm), oscillator strengths (f) and important orbitals involved in transition for F-MOF in their stacked, parallel and diagonal fragments.

\begin{tabular}{|c|c|c|c|c|c|}
\hline $\begin{array}{c}\text { Systems } \\
\text { with } \\
\mathrm{C}_{60}\end{array}$ & $\begin{array}{c}\text { Excited State } \\
\text { (Ex. St.) }\end{array}$ & $\begin{array}{l}\text { Excitation energies } \\
\qquad\left(\mathrm{E}_{0-\mathrm{n}}, \mathrm{eV}\right)\end{array}$ & $\begin{array}{c}\text { Wavelength } \\
\text { (nm) }\end{array}$ & $\begin{array}{c}\text { oscillator } \\
\text { strengths } \\
(f)\end{array}$ & $\begin{array}{c}\text { Important } \\
\text { Orbitals } \\
\text { involved }\end{array}$ \\
\hline \multirow{6}{*}{ Stacked } & 1 & 1.8043 & 687.14 & 0.0060 & $\begin{array}{c}\mathrm{H}-5 \rightarrow \mathrm{L} \\
\mathrm{H}-2 \rightarrow \mathrm{L} \\
\mathrm{H}-1 \rightarrow \mathrm{L} \\
\mathrm{H} \rightarrow \mathrm{L}\end{array}$ \\
\hline & 2 & 1.8235 & 679.93 & 0.0151 & $\begin{array}{c}\mathrm{H}-4 \rightarrow \mathrm{L} \\
\mathrm{H}-1 \rightarrow \mathrm{L} \\
\mathrm{H} \rightarrow \mathrm{L}\end{array}$ \\
\hline & 3 & $1.8 \mathrm{~L}$ & 666.55 & 0.0015 & $\begin{array}{c}\mathrm{H}-1 \rightarrow \mathrm{L}+1 \\
\mathrm{H} \rightarrow \mathrm{L}+1 \\
\mathrm{H} \rightarrow \mathrm{L}+2\end{array}$ \\
\hline & 16 & 2.0724 & H-2.26 & 0.0117 & $\begin{array}{c}\mathrm{H}-6 \rightarrow \mathrm{L} \\
\mathrm{H}-5 \rightarrow \mathrm{L}+2 \\
\mathrm{H}-4 \rightarrow \mathrm{L}+1 \\
\mathrm{H}-4 \rightarrow \mathrm{L}+2\end{array}$ \\
\hline & 26 & 2.2747 & 545.05 & 0.0109 & $\begin{array}{c}\mathrm{H}-8 \rightarrow \mathrm{L}+1 \\
\mathrm{H}-7 \rightarrow \mathrm{L} \\
\mathrm{H}-5 \rightarrow \mathrm{L}+2 \\
\mathrm{H}-2 \rightarrow \mathrm{L}+3 \\
\mathrm{H} \rightarrow \mathrm{L}+4\end{array}$ \\
\hline & 28 & 2.2915 & 541.05 & 0.0139 & $\begin{array}{l}\mathrm{H}-8 \rightarrow \mathrm{L}+1 \\
\mathrm{H}-3 \rightarrow \mathrm{L}+5\end{array}$ \\
\hline
\end{tabular}




\begin{tabular}{|c|c|c|c|c|}
\hline & & & & $\begin{array}{l}\mathrm{H}-2 \rightarrow \mathrm{L}+3 \\
\mathrm{H}-1 \rightarrow \mathrm{L}+3\end{array}$ \\
\hline 42 & 2.5793 & 480.68 & 0.0121 & $\begin{array}{l}\mathrm{H}-5 \rightarrow \mathrm{L}+3 \\
\mathrm{H}-5 \rightarrow \mathrm{L}+4 \\
\mathrm{H}-4 \rightarrow \mathrm{L}+4 \\
\mathrm{H}-3 \rightarrow \mathrm{L}+6\end{array}$ \\
\hline 46 & 2.6729 & 463.86 & 0.0256 & $\begin{array}{l}\mathrm{H}-7 \rightarrow \mathrm{L}+3 \\
\mathrm{H}-5 \rightarrow \mathrm{L}+6 \\
\mathrm{H}-4 \rightarrow \mathrm{L}+6 \\
\mathrm{H}-2 \rightarrow \mathrm{L}+6 \\
\mathrm{H}-1 \rightarrow \mathrm{L}+6\end{array}$ \\
\hline 63 & 3.0501 & 406.49 & 0.0767 & $\begin{array}{c}\mathrm{H}-13 \rightarrow \mathrm{L} \\
\mathrm{H}-9 \rightarrow \mathrm{L}+1 \\
\mathrm{H}-3 \rightarrow \mathrm{L}+7 \\
\mathrm{H}-1 \rightarrow \mathrm{L}+7 \\
\mathrm{H}-1 \rightarrow \mathrm{L}+8 \\
\mathrm{H} \rightarrow \mathrm{L}+7\end{array}$ \\
\hline
\end{tabular}

S2.3. Results for $\mathrm{C}_{60}$ incorporated H_DA-C60 in Type-I interface. 


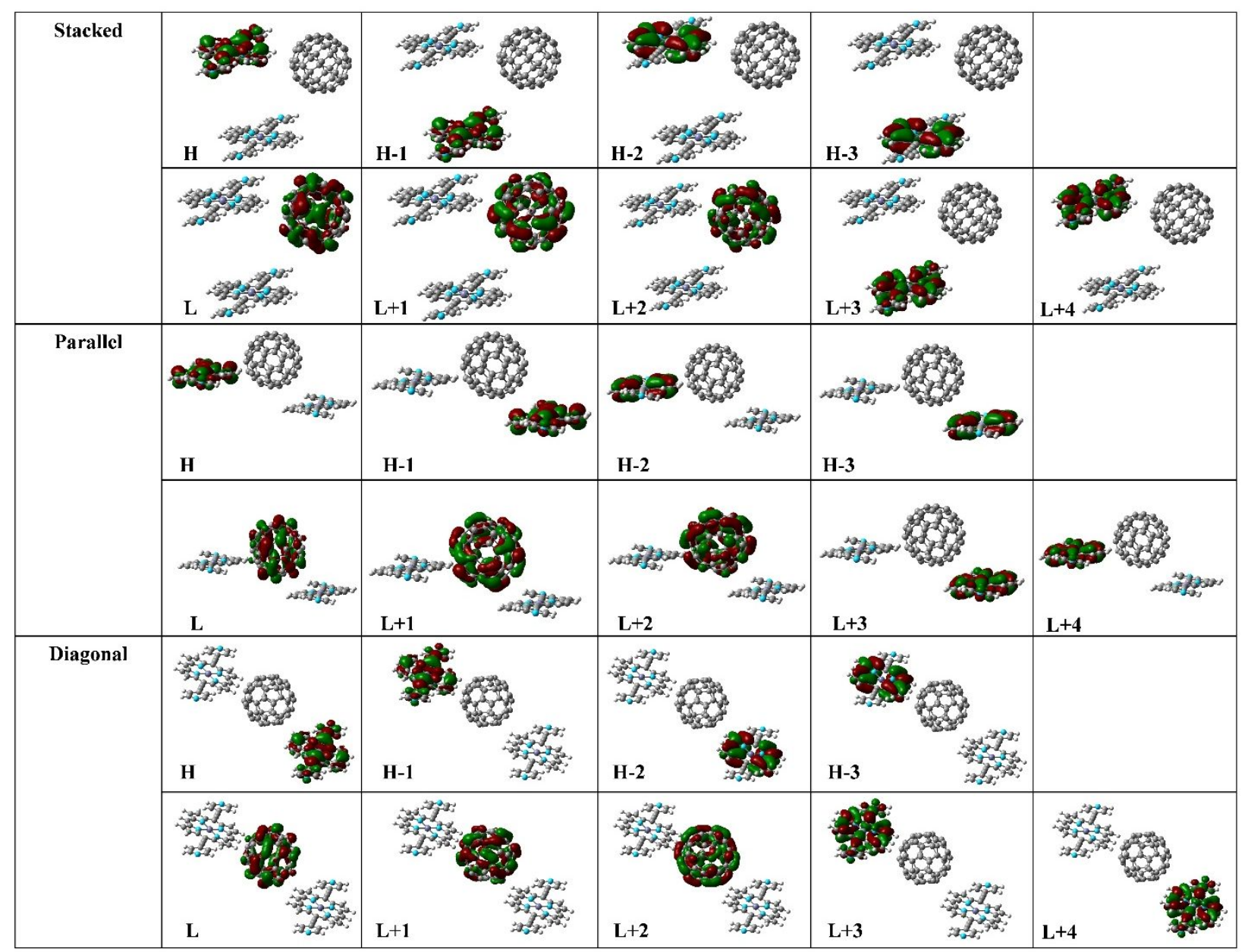

Figure S17. Frontier molecular orbitals for Type-I interface of H_DA-MOF in stacked, parallel and diagonal fragments.

TableS4. Excited State, excitation energies (eV), wavelength (nm), oscillator strengths (f) and important orbitals involved in transition for Type-I interfaces of H_DA-MOF in their stacked, parallel and diagonal fragments.

\begin{tabular}{|l|c|c|c|c|c|}
\hline Systems & Excited State & Excitation energies & Wavelength & Oscillator & Important \\
sith $\mathrm{C}_{60}$ & $($ Ex. St. $)$ & $\left(\mathrm{E}_{0-\mathrm{n}}, \mathrm{eV}\right)$ & $(\mathrm{nm})$ & $(f)$ & Orbitals \\
& & & & involved \\
\hline
\end{tabular}




\begin{tabular}{|c|c|c|c|c|c|}
\hline \multirow{4}{*}{ Stack } & 26 & 2.1539 & 575.63 & 1.0009 & $\begin{array}{l}\mathrm{H}-1 \rightarrow \mathrm{L}+3 \\
\mathrm{H} \rightarrow \mathrm{L}+4\end{array}$ \\
\hline & 27 & 2.1957 & 564.67 & 0.0062 & $\begin{array}{l}\mathrm{H}-3 \rightarrow \mathrm{L}+3 \\
\mathrm{H}-1 \rightarrow \mathrm{L}+5\end{array}$ \\
\hline & 28 & 2.1978 & 564.13 & 0.0145 & $\begin{array}{c}\mathrm{H}-2 \rightarrow \mathrm{L}+4 \\
\mathrm{H} \rightarrow \mathrm{L}+6\end{array}$ \\
\hline & 71 & 3.0654 & 404.47 & 0.5409 & $\begin{array}{l}\mathrm{H}-3 \rightarrow \mathrm{L}+3 \\
\mathrm{H}-2 \rightarrow \mathrm{L}+4 \\
\mathrm{H}-1 \rightarrow \mathrm{L}+5\end{array}$ \\
\hline \multirow{5}{*}{ Parallel } & 25 & 2.1370 & 580.18 & 0.0139 & $\begin{array}{c}\mathrm{H}-1 \rightarrow \mathrm{L}+3 \\
\mathrm{H} \rightarrow \mathrm{L}+4\end{array}$ \\
\hline & 26 & 2.1503 & 576.60 & 1.0369 & $\begin{array}{c}\mathrm{H}-1 \rightarrow \mathrm{L}+3 \\
\mathrm{H} \rightarrow \mathrm{L}+4\end{array}$ \\
\hline & 27 & 2.1972 & 564.29 & 0.0144 & $\begin{array}{l}\mathrm{H}-3 \rightarrow \mathrm{L}+3 \\
\mathrm{H}-1 \rightarrow \mathrm{L}+5\end{array}$ \\
\hline & 68 & 3.0483 & 406.73 & 1.0931 & $\begin{array}{l}\mathrm{H}-3 \rightarrow \mathrm{L}+3 \\
\mathrm{H}-2 \rightarrow \mathrm{L}+4 \\
\mathrm{H}-1 \rightarrow \mathrm{L}+5\end{array}$ \\
\hline & 92 & 3.1258 & 396.65 & 3.9501 & $\begin{array}{l}\mathrm{H}-3 \rightarrow \mathrm{L}+5 \\
\mathrm{H}-2 \rightarrow \mathrm{L}+6\end{array}$ \\
\hline
\end{tabular}




\begin{tabular}{|c|c|c|c|c|c|}
\hline \multirow{5}{*}{ Diagonal } & 25 & 2.1411 & 579.06 & 0.0033 & $\begin{array}{c}\mathrm{H}-1 \rightarrow \mathrm{L}+3 \\
\mathrm{H} \rightarrow \mathrm{L}+4\end{array}$ \\
\hline & 26 & 2.1495 & 576.80 & 1.0600 & $\begin{array}{c}\mathrm{H}-1 \rightarrow \mathrm{L}+3 \\
\mathrm{H} \rightarrow \mathrm{L}+4\end{array}$ \\
\hline & 27 & 2.1980 & 564.08 & 0.0236 & $\begin{array}{l}\mathrm{H}-3 \rightarrow \mathrm{L}+3 \\
\mathrm{H}-2 \rightarrow \mathrm{L}+4 \\
\mathrm{H}-1 \rightarrow \mathrm{L}+5\end{array}$ \\
\hline & 77 & 3.0508 & 406.40 & 0.4824 & $\begin{array}{l}\mathrm{H}-13 \rightarrow \mathrm{L}+1 \\
\mathrm{H}-2 \rightarrow \mathrm{L}+4 \\
\mathrm{H}-1 \rightarrow \mathrm{L}+5\end{array}$ \\
\hline & 78 & 3.0512 & 406.34 & 0.5605 & $\begin{array}{l}\mathrm{H}-2 \rightarrow \mathrm{L}+4 \\
\mathrm{H}-1 \rightarrow \mathrm{L}+5 \\
\mathrm{H} \rightarrow \mathrm{L}+6\end{array}$ \\
\hline
\end{tabular}

S2.4. Results for $\mathrm{C}_{60}$ incorporated H_DA-C60 in Type-II interface. 


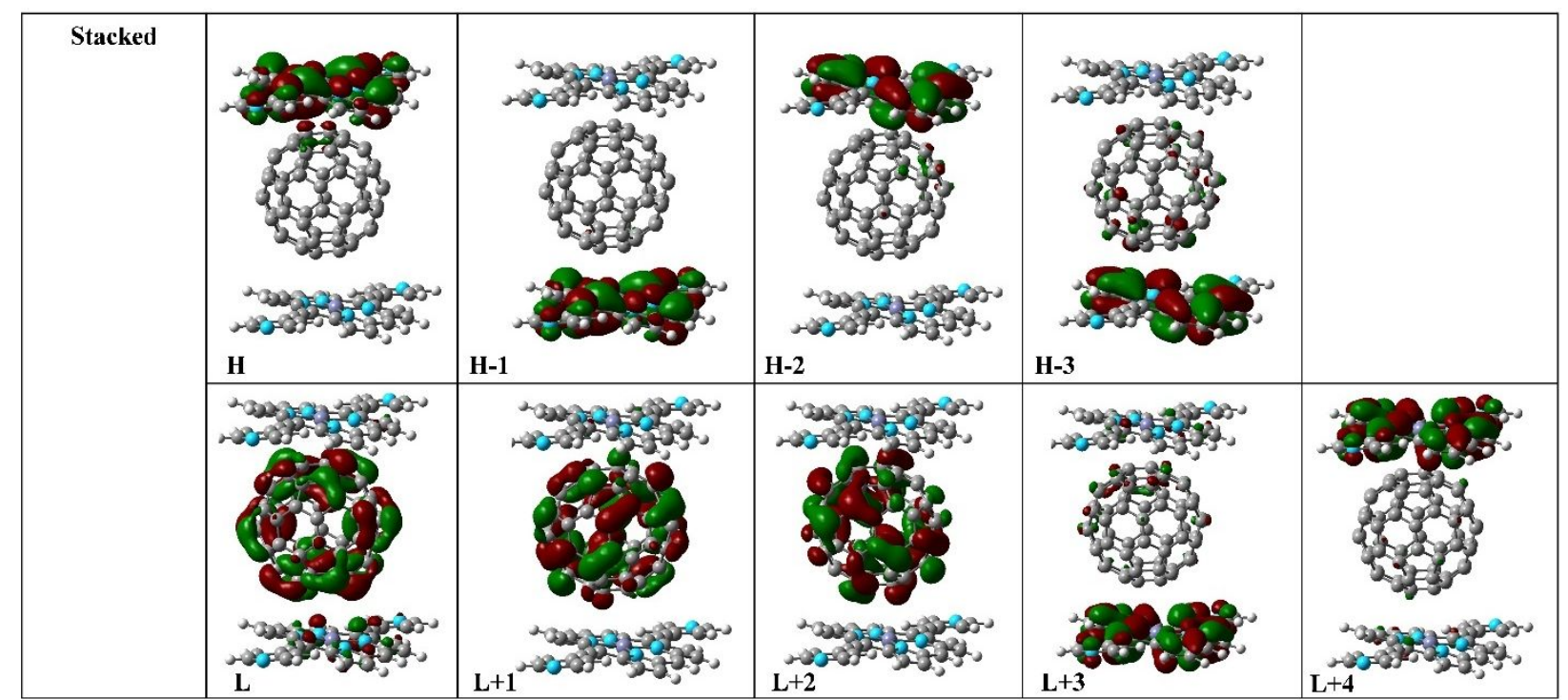

Figure S18. Frontier molecular orbitals for Type-II interface of H_DA-MOF in stacked fragment.

TableS5. Excited State, excitation energies (eV), wavelength (nm), oscillator strengths (f) and important orbitals involved in transition for Type-II interfaces of H_DA-MOF in their stacked fragment.

\begin{tabular}{|c|c|c|c|c|c|}
\hline $\begin{array}{c}\text { Systems } \\
\text { with } \\
\mathrm{C}_{60}\end{array}$ & $\begin{array}{c}\text { Excited } \\
\text { State }(\text { Ex. } \\
\text { St.) }\end{array}$ & $\begin{array}{c}\text { Excitation energies } \\
\left(\mathrm{E}_{0-\mathrm{n}}, \mathrm{eV}\right)\end{array}$ & $\begin{array}{c}\text { Wavelength } \\
(\mathrm{nm})\end{array}$ & $\begin{array}{c}\text { Oscillator } \\
\text { strengths }(f)\end{array}$ & $\begin{array}{c}\text { Important Orbitals } \\
\text { involved }\end{array}$ \\
\hline \multirow{2}{*}{ Stack } & 1 & 1.6171 & 766.70 & 0.0020 & $\mathrm{H} \rightarrow \mathrm{L}$ \\
\cline { 2 - 6 } & 2 & 1.6517 & 750.63 & 0.0081 & $\mathrm{H}-3 \rightarrow \mathrm{L}+1$ \\
\cline { 2 - 6 } & 4 & 1.7144 & 723.18 & 0.0168 & $\mathrm{H}-1 \rightarrow \mathrm{L}+2$ \\
& & & & & $\mathrm{H}-1 \rightarrow \mathrm{L}$ \\
\hline
\end{tabular}




\begin{tabular}{|c|c|c|c|c|}
\hline 8 & 1.9273 & 643.31 & 0.0121 & $\mathrm{H}-2 \rightarrow \mathrm{L}$ \\
\hline 22 & 2.1240 & 583.73 & 0.1494 & $\begin{array}{l}\mathrm{H}-6 \rightarrow \mathrm{L}+1 \\
\mathrm{H}-1 \rightarrow \mathrm{L}+3\end{array}$ \\
\hline 78 & 3.0741 & 403.32 & 1.3061 & $\begin{array}{l}\mathrm{H}-10 \rightarrow \mathrm{L}+3 \\
\mathrm{H}-8 \rightarrow \mathrm{L}+6 \\
\mathrm{H}-2 \rightarrow \mathrm{L}+6\end{array}$ \\
\hline
\end{tabular}

S2.5. Results for $\mathrm{C}_{60}$ incorporated H_DA-C60 in Type-III interface. 


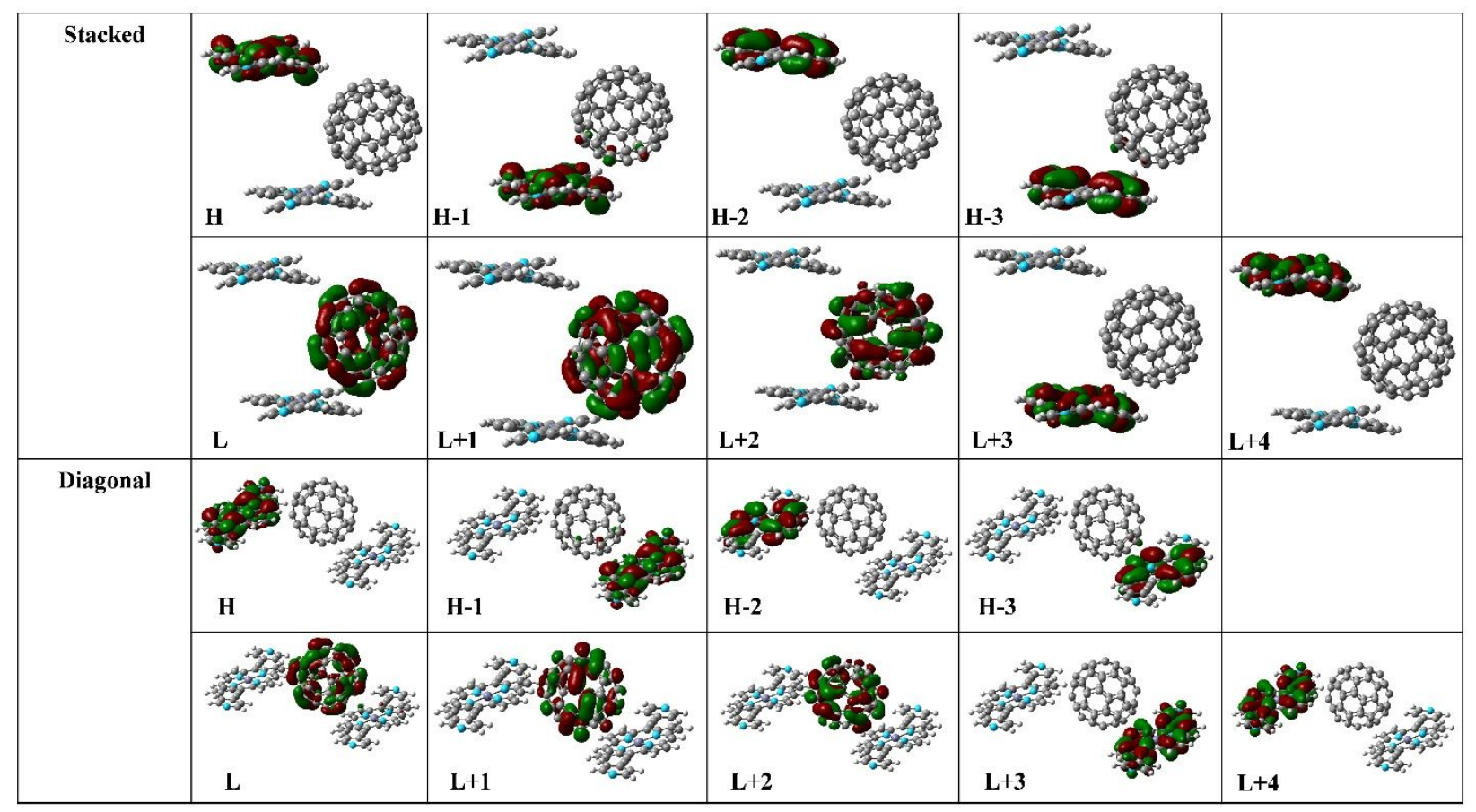

Figure S19. Frontier molecular orbitals for Type-III interface of H_DA-MOF in stacked and diagonal fragments.

TableS6. Excited State, excitation energies (eV), wavelength (nm), oscillator strengths (f) and important orbitals involved in transition for Type-III interfaces of H_DA-MOF in their stacked and diagonal fragments.

\begin{tabular}{|c|l|l|l|l|c|}
\hline $\begin{array}{c}\text { Systems } \\
\text { with } \mathrm{C}_{60}\end{array}$ & $\begin{array}{c}\text { Excited State } \\
(\text { Ex. St. })\end{array}$ & $\begin{array}{c}\text { Excitation energies } \\
\left(\mathrm{E}_{0-\mathrm{n}}, \mathrm{eV}\right)\end{array}$ & $\begin{array}{c}\text { Wavelength } \\
(\mathrm{nm})\end{array}$ & $\begin{array}{c}\text { Oscillator } \\
\text { strengths } \\
(f)\end{array}$ & $\begin{array}{c}\text { Important } \\
\text { Orbitals } \\
\text { involved }\end{array}$ \\
\hline \multirow{3}{*}{ Stacked } & 1 & 1.4761 & 839.97 & $\mathrm{f}=0.0004$ & $\mathrm{H} \rightarrow \mathrm{L}$ \\
\cline { 2 - 6 } & 2 & 1.4888 & 832.76 & $\mathrm{f}=0.0012$ & $\mathrm{H} \rightarrow \mathrm{L}+1$ \\
\cline { 2 - 6 } & 4 & 1.5402 & 804.98 & 0.0021 & $\mathrm{H}-1 \rightarrow \mathrm{L}$ \\
\hline
\end{tabular}




\begin{tabular}{|c|c|c|c|c|c|}
\hline & & & & & $\mathrm{H}-1 \rightarrow \mathrm{L}+1$ \\
\hline & 5 & 1.5625 & 793.51 & 0.036 & $\begin{array}{c}\mathrm{H}-1 \rightarrow \mathrm{L} \\
\mathrm{H}-1 \rightarrow \mathrm{L}+1\end{array}$ \\
\hline & 9 & 1.7830 & 695.38 & 0.0020 & $\mathrm{H}-2 \rightarrow \mathrm{L}+2$ \\
\hline & 11 & 1.8385 & 674.38 & 0.0052 & $\mathrm{H}-3 \rightarrow \mathrm{L}+1$ \\
\hline & 13 & 1.8831 & 658.42 & 0.0212 & $\begin{array}{l}\mathrm{H}-13 \rightarrow \mathrm{L} \\
\mathrm{H}-3 \rightarrow \mathrm{L}+2\end{array}$ \\
\hline & 27 & 2.1501 & 576.65 & 0.5204 & $\begin{array}{c}\mathrm{H}-2 \rightarrow \mathrm{L}+3 \\
\mathrm{H}-2 \rightarrow \mathrm{L}+6 \\
\mathrm{H} \rightarrow \mathrm{L}+4\end{array}$ \\
\hline & 87 & 3.1006 & 399.88 & 1.0957 & $\begin{array}{c}\mathrm{H}-2 \rightarrow \mathrm{L}+6 \\
\mathrm{H} \rightarrow \mathrm{L}+4\end{array}$ \\
\hline & 1 & 1.3963 & 887.94 & 0.0004 & $\mathrm{H} \rightarrow \mathrm{L}$ \\
\hline & 2 & 1.4109 & 878.77 & $\mathrm{f}=0.0008$ & $\begin{array}{l}H \rightarrow L+1 \\
H->467\end{array}$ \\
\hline Diagonal & 4 & 1.5361 & 807.11 & 0.0019 & $\begin{array}{c}\mathrm{H}-1 \rightarrow \mathrm{L} \\
\mathrm{H}-1 \rightarrow \mathrm{L}+1 \\
\mathrm{H}-1 \rightarrow \mathrm{L}+2\end{array}$ \\
\hline & 5 & 801.97 & 801.97 & 0.0470 & $\mathrm{H}-1 \rightarrow \mathrm{L}$ \\
\hline
\end{tabular}




\begin{tabular}{|c|c|c|c|c|}
\hline & & & & $\mathrm{H}-1 \rightarrow \mathrm{L}+1$ \\
\hline 13 & 1.8547 & 668.50 & 0.0281 & $\begin{array}{c}\mathrm{H}-3 \rightarrow \mathrm{L} \\
\mathrm{H}-3 \rightarrow \mathrm{L}+1 \\
\mathrm{H}-3 \rightarrow \mathrm{L}+2\end{array}$ \\
\hline 27 & 2.1486 & 577.04 & 0.5523 & $\begin{array}{r}\mathrm{H}-2 \rightarrow \mathrm{L}+6 \\
\mathrm{H}-2 \rightarrow \mathrm{L}+4 \\
\mathrm{H} \rightarrow \mathrm{L}+4\end{array}$ \\
\hline 95 & 3.1047 & 399.34 & 1.2467 & $\begin{aligned} \mathrm{H}-2 \rightarrow \mathrm{L}+6 \\
\mathrm{H} \rightarrow \mathrm{L}+4\end{aligned}$ \\
\hline
\end{tabular}

S2.6. Results for $\mathrm{C}_{60}$ incorporated H_F-C60 in Type-A interface.

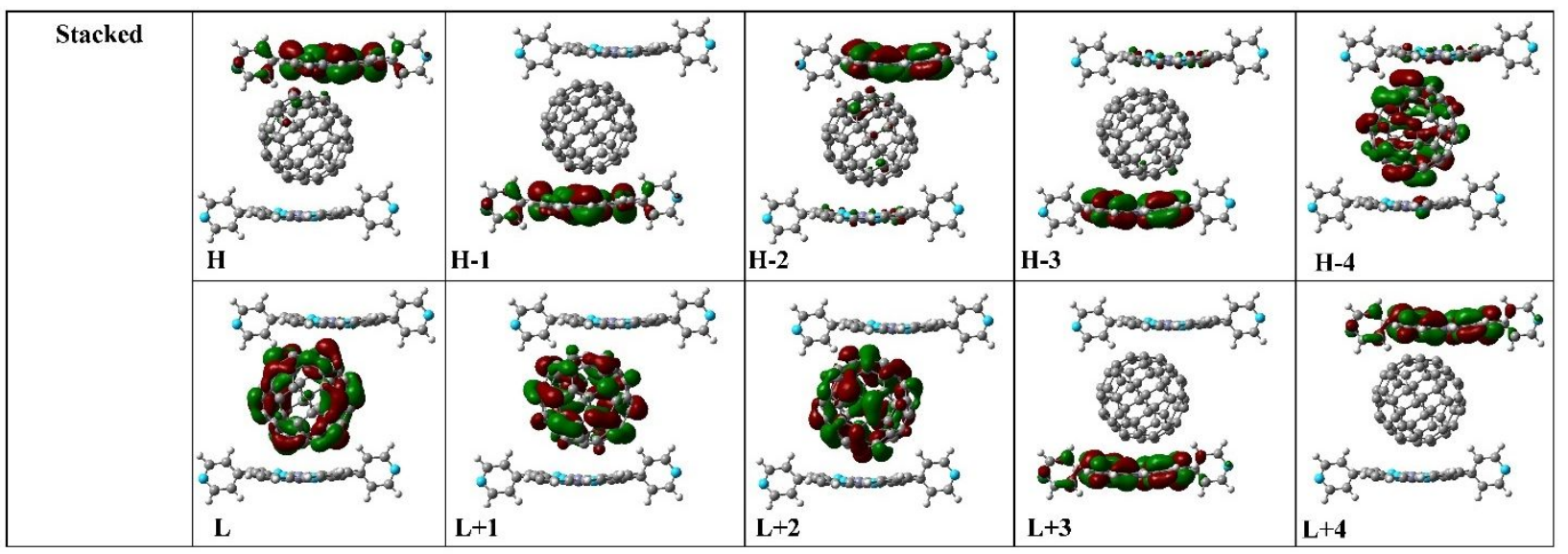

Figure S20. Frontier molecular orbitals for Type-A interface of H_F-MOF in stacked fragment. 
TableS7. Excited State, excitation energies (eV), wavelength (nm), oscillator strengths (f) and important orbitals involved in transition for Type-A interfaces of H_F-MOF in their stacked fragments.

\begin{tabular}{|c|c|c|c|c|c|}
\hline $\begin{array}{c}\text { Systems } \\
\text { with } \\
\mathrm{C}_{60}\end{array}$ & $\begin{array}{l}\text { Excited State } \\
\text { (Ex. St.) }\end{array}$ & $\begin{array}{c}\text { Excitation energies } \\
\qquad\left(\mathrm{E}_{0-\mathrm{n}}, \mathrm{eV}\right)\end{array}$ & $\begin{array}{l}\text { Wavelength } \\
\text { (nm) }\end{array}$ & $\begin{array}{c}\text { Oscillator } \\
\text { strengths } \\
(f)\end{array}$ & $\begin{array}{c}\text { Important } \\
\text { Orbitals } \\
\text { involved }\end{array}$ \\
\hline \multirow{7}{*}{ Stacked } & 1 & 1.6970 & 730.61 & 0.0230 & $\begin{array}{c}\mathrm{H}-2 \rightarrow \mathrm{L} \\
\mathrm{H} \rightarrow \mathrm{L}\end{array}$ \\
\hline & 2 & 1.7254 & 718.57 & 0.0033 & $\begin{array}{c}\mathrm{H}-1 \rightarrow \mathrm{L} \\
\mathrm{H}-1 \rightarrow \mathrm{L}+1 \\
\mathrm{H} \rightarrow \mathrm{L}+1\end{array}$ \\
\hline & 5 & 1.7612 & 703.98 & 0.0134 & $\begin{array}{c}\mathrm{H}-2 \rightarrow \mathrm{L} \\
\mathrm{H} \rightarrow \mathrm{L}\end{array}$ \\
\hline & 12 & 1.8604 & 666.43 & 0.0169 & $\begin{array}{l}\mathrm{H}-3 \rightarrow \mathrm{L}+1 \\
\mathrm{H}-3 \rightarrow \mathrm{L}+2\end{array}$ \\
\hline & 28 & 2.3610 & 525.14 & 0.0071 & $\begin{array}{c}\mathrm{H}-2 \rightarrow \mathrm{L}+4 \\
\mathrm{H}-2 \rightarrow \mathrm{L}+5 \\
\mathrm{H}-2 \rightarrow \mathrm{L}+6 \\
\mathrm{H} \rightarrow \mathrm{L}+4 \\
\mathrm{H} \rightarrow \mathrm{L}+5\end{array}$ \\
\hline & 43 & 2.8362 & H-3.15 & 0.0290 & $\begin{array}{l}\mathrm{H}-5 \rightarrow \mathrm{L}+4 \\
\mathrm{H}-4 \rightarrow \mathrm{L}+5 \\
\mathrm{H}-4 \rightarrow \mathrm{L}+6 \\
\mathrm{H}-2 \rightarrow \mathrm{L}+6\end{array}$ \\
\hline & 51 & 2.9417 & 421.47 & 0.0649 & $\begin{array}{l}\mathrm{H}-6 \rightarrow \mathrm{L}+5 \\
\mathrm{H}-3 \rightarrow \mathrm{L}+6\end{array}$ \\
\hline
\end{tabular}




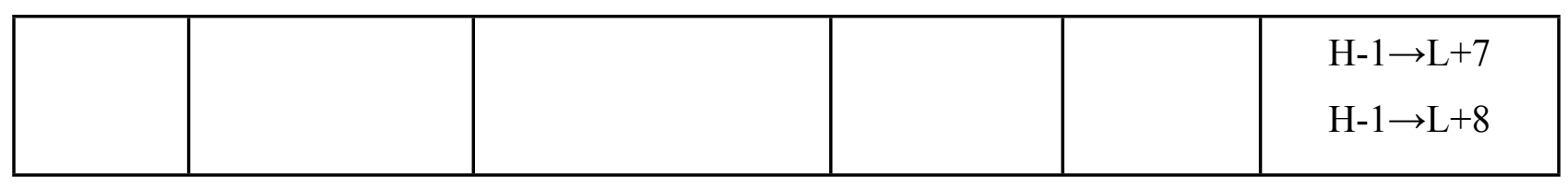

S2.7. Results for $\mathrm{C}_{60}$ incorporated H_F-C60 in Type-B interface.

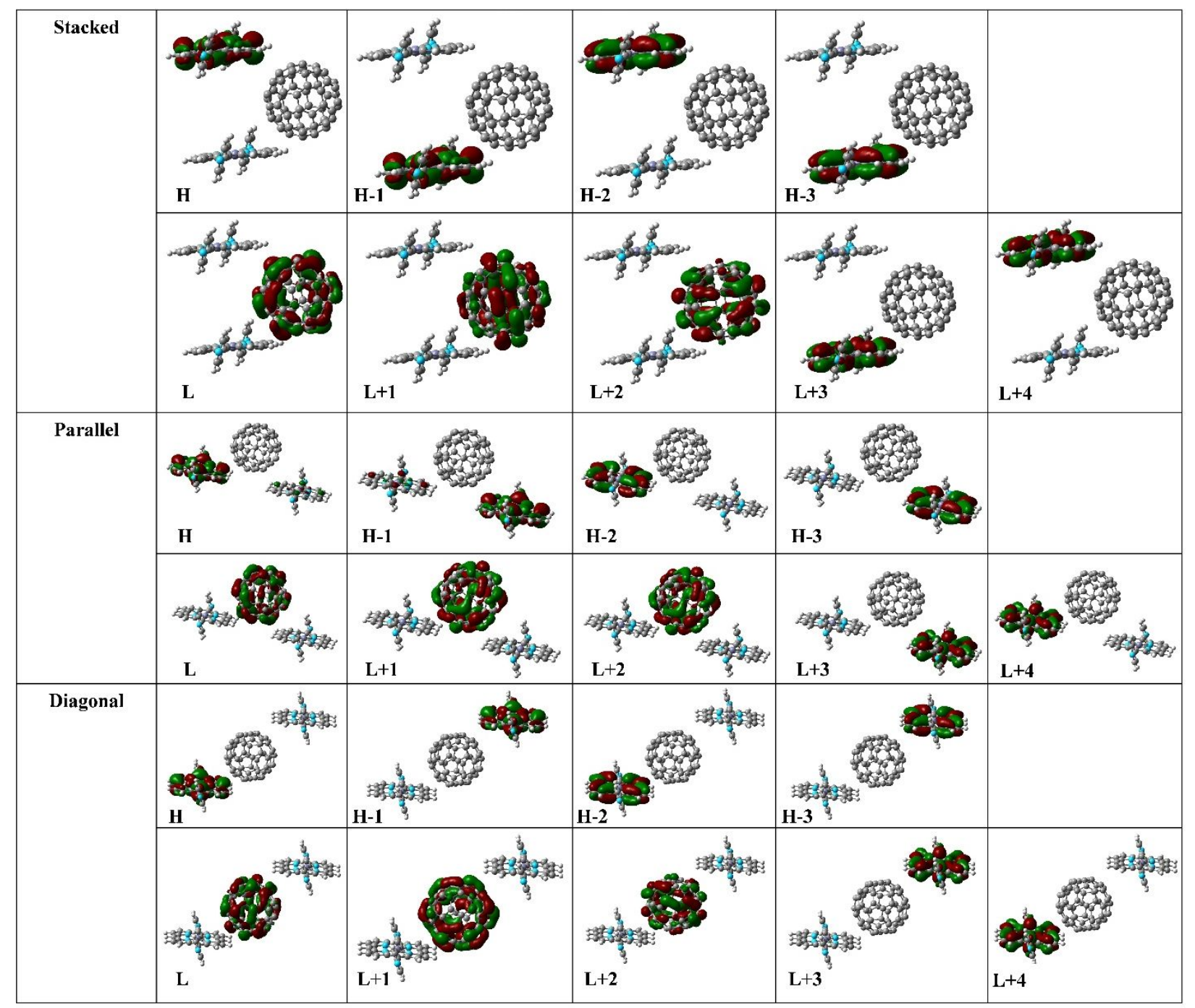

Figure S21. Frontier molecular orbitals for Type-B interface of H_DA-MOF in stacked, parallel and diagonal fragments. 
TableS8. Excited State, excitation energies (eV), wavelength (nm), oscillator strengths (f) and important orbitals involved in transition for Type-B interfaces of H_F-MOF in their stacked, parallel and diagonal fragments.

\begin{tabular}{|c|c|c|c|c|c|}
\hline $\begin{array}{l}\text { Systems } \\
\text { with } \mathrm{C}_{60}\end{array}$ & $\begin{array}{c}\text { Excited State } \\
\text { (Ex. St.) }\end{array}$ & $\begin{array}{l}\text { Excitation energies } \\
\qquad\left(\mathrm{E}_{0-\mathrm{n}}, \mathrm{eV}\right)\end{array}$ & $\begin{array}{c}\text { Wavelength } \\
(\mathrm{nm})\end{array}$ & $\begin{array}{c}\text { Oscillator } \\
\text { strengths } \\
(f)\end{array}$ & $\begin{array}{c}\text { Important } \\
\text { Orbitals } \\
\text { involved }\end{array}$ \\
\hline \multirow{5}{*}{ Stacked } & 1 & $1.5 \mathrm{H}$ & 803.00 & 0.0010 & $\begin{array}{c}\mathrm{H}-1 \rightarrow \mathrm{L} \\
\mathrm{H} \rightarrow \mathrm{L} \\
\mathrm{H} \rightarrow \mathrm{L}+1\end{array}$ \\
\hline & 2 & 1.5448 & 802.58 & 0.0070 & $\begin{array}{c}\mathrm{H}-1 \rightarrow \mathrm{L} \\
\mathrm{H}-1 \rightarrow \mathrm{L}+1 \\
\mathrm{H} \rightarrow \mathrm{L}\end{array}$ \\
\hline & 8 & 1.6127 & 768.81 & 0.0027 & $\begin{array}{c}\mathrm{H}-3 \rightarrow \mathrm{L} \\
\mathrm{H}-2 \rightarrow \mathrm{L} \\
\mathrm{H}-2 \rightarrow \mathrm{L}+1\end{array}$ \\
\hline & 29 & 2.4135 & 513.70 & 0.0059 & $\begin{array}{c}\mathrm{H}-2 \rightarrow \mathrm{L}+4 \\
\mathrm{H}-2 \rightarrow \mathrm{L}+6 \\
\mathrm{H} \rightarrow \mathrm{L}+4 \\
\mathrm{H} \rightarrow \mathrm{L}+6\end{array}$ \\
\hline & 40 & 2.7579 & 449.56 & 0.0142 & $\begin{array}{l}\mathrm{H}-1 \rightarrow \mathrm{L}+5 \\
\mathrm{H}-1 \rightarrow \mathrm{L}+7 \\
\mathrm{H}-1 \rightarrow \mathrm{L}+8 \\
\mathrm{H}-1 \rightarrow \mathrm{L}+9\end{array}$ \\
\hline Parallel & 1 & 1.5641 & 792.67 & 0.0072 & $\begin{array}{c}\mathrm{H}-1 \rightarrow \mathrm{L} \\
\mathrm{H} \rightarrow \mathrm{L}\end{array}$ \\
\hline
\end{tabular}




\begin{tabular}{|c|c|c|c|c|c|}
\hline & 3 & 1.5736 & 787.91 & 0.0034 & $\begin{array}{c}\mathrm{H}-1 \rightarrow \mathrm{L}+1 \\
\mathrm{H}-1 \rightarrow \mathrm{L}+2 \\
\mathrm{H} \rightarrow \mathrm{L}+1\end{array}$ \\
\hline & 24 & 2.2603 & 548.52 & 0.0020 & $\begin{array}{c}\mathrm{H}-8 \rightarrow \mathrm{L} \\
\mathrm{H}-8 \rightarrow \mathrm{L}+1 \\
\mathrm{H}-6 \rightarrow \mathrm{L} \\
\mathrm{H}-5 \rightarrow \mathrm{L} \\
\mathrm{H}-4 \rightarrow \mathrm{L}+2\end{array}$ \\
\hline & 54 & 2.9054 & 426.74 & 0.0058 & $\begin{array}{c}\mathrm{H}-10 \rightarrow \mathrm{L}+1 \\
\mathrm{H}-9 \rightarrow \mathrm{L}+1\end{array}$ \\
\hline & 62 & 2.9482 & 420.54 & 0.0048 & $\begin{array}{c}\mathrm{H}-12 \rightarrow \mathrm{L} \\
\mathrm{H}-12 \rightarrow \mathrm{L}+1 \\
\mathrm{H}-12 \rightarrow \mathrm{L}+2 \\
\mathrm{H}-11 \rightarrow \mathrm{L}+2\end{array}$ \\
\hline \multirow{5}{*}{ Diagonal } & 2 & 1.5746 & 787.40 & 0.0008 & $\mathrm{H}-1 \rightarrow \mathrm{L}$ \\
\hline & 6 & 1.5944 & 777.62 & 0.0028 & $\mathrm{H}-1 \rightarrow \mathrm{L}+2$ \\
\hline & 28 & 2.4145 & 513.49 & 0.0053 & $\begin{array}{l}\mathrm{H}-3 \rightarrow \mathrm{L}+3 \\
\mathrm{H}-3 \rightarrow \mathrm{L}+5 \\
\mathrm{H}-1 \rightarrow \mathrm{L}+3 \\
\mathrm{H}-1 \rightarrow \mathrm{L}+5\end{array}$ \\
\hline & 31 & 2.4232 & 511.66 & 0.0073 & $\begin{array}{c}\mathrm{H}-2 \rightarrow \mathrm{L}+4 \\
\mathrm{H}-2 \rightarrow \mathrm{L}+6 \\
\mathrm{H} \rightarrow \mathrm{L}+4 \\
\mathrm{H} \rightarrow \mathrm{L}+6\end{array}$ \\
\hline & 41 & 2.7918 & 444.10 & 0.0021 & $\begin{array}{l}\mathrm{H}-1 \rightarrow \mathrm{L}+7 \\
\mathrm{H}-1 \rightarrow \mathrm{L}+8\end{array}$ \\
\hline
\end{tabular}


S2.8. Results for $\mathrm{C}_{60}$ incorporated $\mathrm{NH}_{2}$ DA-C60 in Type-I interface.

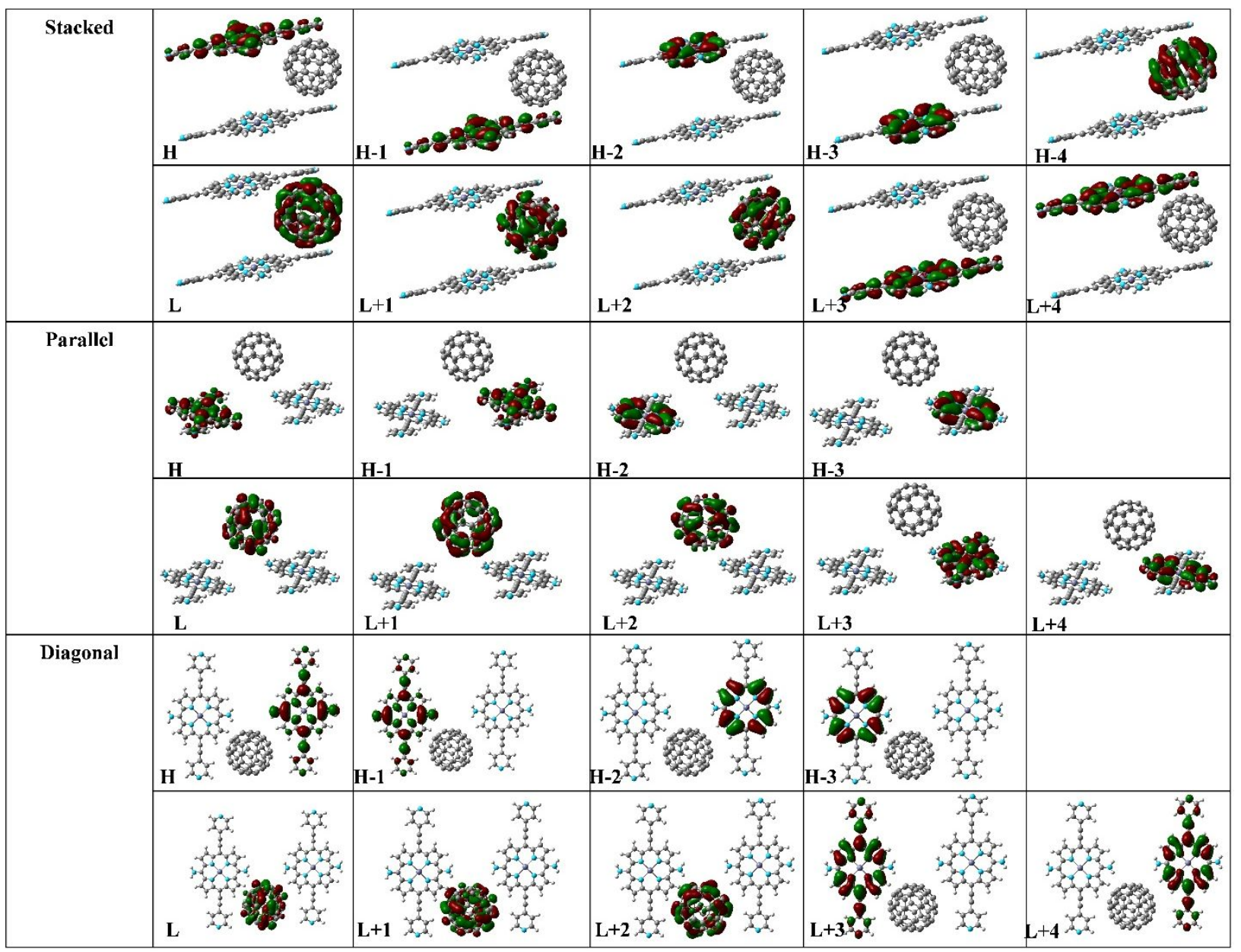

Figure S22. Frontier molecular orbitals for Type-I interface of $\mathrm{NH}_{2}$ DA-MOF in stacked, parallel and diagonal fragments.

TableS9. Excited State, excitation energies (eV), wavelength (nm), oscillator strengths (f) and important orbitals involved in transition for Type-I interfaces of $\mathrm{NH}_{2}$ DA-MOF in their stacked, parallel and diagonal fragments.

\begin{tabular}{|l|c|c|c|c|c|}
\hline Systems & Excited State & Excitation energies & Wavelength & Oscillator & Important \\
with $\mathrm{C}_{60}$ & $($ Ex. St. $)$ & $\left(\mathrm{E}_{0-\mathrm{n}}, \mathrm{eV}\right)$ & $(\mathrm{nm})$ & $\begin{array}{c}\text { Orengths } \\
(f)\end{array}$ & involved \\
\hline
\end{tabular}




\begin{tabular}{|c|c|c|c|c|c|}
\hline \multirow{4}{*}{ Stacked } & 9 & 1.6345 & 758.56 & 0.0014 & $\begin{array}{c}\mathrm{H}-3 \rightarrow \mathrm{L}+8 \\
\mathrm{H}-2 \rightarrow \mathrm{L}+9 \\
\mathrm{H}-1 \rightarrow \mathrm{L}+3 \\
\mathrm{H} \rightarrow \mathrm{L}+4\end{array}$ \\
\hline & 13 & 1.6778 & 738.95 & 1.2978 & $\begin{array}{c}\mathrm{H}-3 \rightarrow \mathrm{L}+8 \\
\mathrm{H}-2 \rightarrow \mathrm{L}+9 \\
\mathrm{H}-1 \rightarrow \mathrm{L}+3 \\
\mathrm{H} \rightarrow \mathrm{L}+4\end{array}$ \\
\hline & 35 & 2.1637 & 573.03 & 0.0026 & $\begin{array}{c}\mathrm{H}-3 \rightarrow \mathrm{L}+3 \\
\mathrm{H}-2 \rightarrow \mathrm{L}+4 \\
\mathrm{H}-1 \rightarrow \mathrm{L}+8 \\
\mathrm{H} \rightarrow \mathrm{L}+9\end{array}$ \\
\hline & 88 & 2.9313 & 422.96 & 0.0002 & $\mathrm{H}-18 \rightarrow \mathrm{L}+1$ \\
\hline \multirow{4}{*}{ Parallel } & 9 & $1.6 \mathrm{H}-11$ & 752.84 & 0.0078 & $\begin{array}{c}\mathrm{H}-2 \rightarrow \mathrm{L}+9 \\
\mathrm{H}-1 \rightarrow \mathrm{L}+3 \\
\mathrm{H} \rightarrow \mathrm{L}+4\end{array}$ \\
\hline & 10 & 1.6697 & 742.57 & 1.3263 & $\begin{array}{c}\mathrm{H}-3 \rightarrow \mathrm{L}+8 \\
\mathrm{H}-2 \rightarrow \mathrm{L}+9 \\
\mathrm{H}-1 \rightarrow \mathrm{L}+3 \\
\mathrm{H} \rightarrow \mathrm{L}+4\end{array}$ \\
\hline & 33 & 2.1628 & 573.27 & 0.0033 & $\begin{array}{c}\mathrm{H}-2 \rightarrow \mathrm{L}+4 \\
\mathrm{H}-1 \rightarrow \mathrm{L}+8 \\
\mathrm{H} \rightarrow \mathrm{L}+9\end{array}$ \\
\hline & 96 & 2.9734 & 416.98 & 0.1335 & $\begin{array}{c}\mathrm{H}-11 \rightarrow \mathrm{L}+4 \\
\mathrm{H}-3 \rightarrow \mathrm{L}+3 \\
\mathrm{H}-2 \rightarrow \mathrm{L}+4 \\
\mathrm{H} \rightarrow \mathrm{L}+9\end{array}$ \\
\hline
\end{tabular}




\begin{tabular}{|c|c|c|c|c|c|}
\hline & 98 & 2.9754 & 416.70 & 1.0204 & $\begin{array}{c}\mathrm{H}-12 \rightarrow \mathrm{L}+3 \\
\mathrm{H}-11 \rightarrow \mathrm{L}+4 \\
\mathrm{H}-3 \rightarrow \mathrm{L}+3 \\
\mathrm{H}-2 \rightarrow \mathrm{L}+4 \\
\mathrm{H}-1 \rightarrow \mathrm{L}+8 \\
\mathrm{H} \rightarrow \mathrm{L}+9\end{array}$ \\
\hline \multirow{5}{*}{ Diagonal } & 15 & $1.6 \mathrm{~L}+10$ & 751.83 & 0.0039 & $\begin{array}{c}\mathrm{H}-3 \rightarrow \mathrm{L}+8 \\
\mathrm{H}-2 \rightarrow \mathrm{L}+9 \\
\mathrm{H}-1 \rightarrow \mathrm{L}+3 \\
\mathrm{H} \rightarrow \mathrm{L}+4\end{array}$ \\
\hline & 16 & 1.6653 & 744.54 & 1.3575 & $\begin{array}{c}\mathrm{H}-3 \rightarrow \mathrm{L}+8 \\
\mathrm{H}-2 \rightarrow \mathrm{L}+9 \\
\mathrm{H}-1 \rightarrow \mathrm{L}+3 \\
\mathrm{H} \rightarrow \mathrm{L}+4\end{array}$ \\
\hline & 33 & 2.1634 & 573.11 & 0.0036 & $\begin{array}{c}\mathrm{H}-3 \rightarrow \mathrm{L}+3 \\
\mathrm{H}-2 \rightarrow \mathrm{L}+4 \\
\mathrm{H}-1 \rightarrow \mathrm{L}+8 \\
\mathrm{H} \rightarrow \mathrm{L}+9\end{array}$ \\
\hline & 97 & 2.9120 & 425.78 & 0.0043 & $\mathrm{H}-1 \rightarrow \mathrm{L}+12$ \\
\hline & 98 & 2.9132 & 425.59 & 0.0045 & $\mathrm{H} \rightarrow \mathrm{L}+13$ \\
\hline
\end{tabular}


S2.9. Results for $\mathrm{C}_{60}$ incorporated $\mathrm{NH}_{2}$ DA-C60 in Type-II interface.

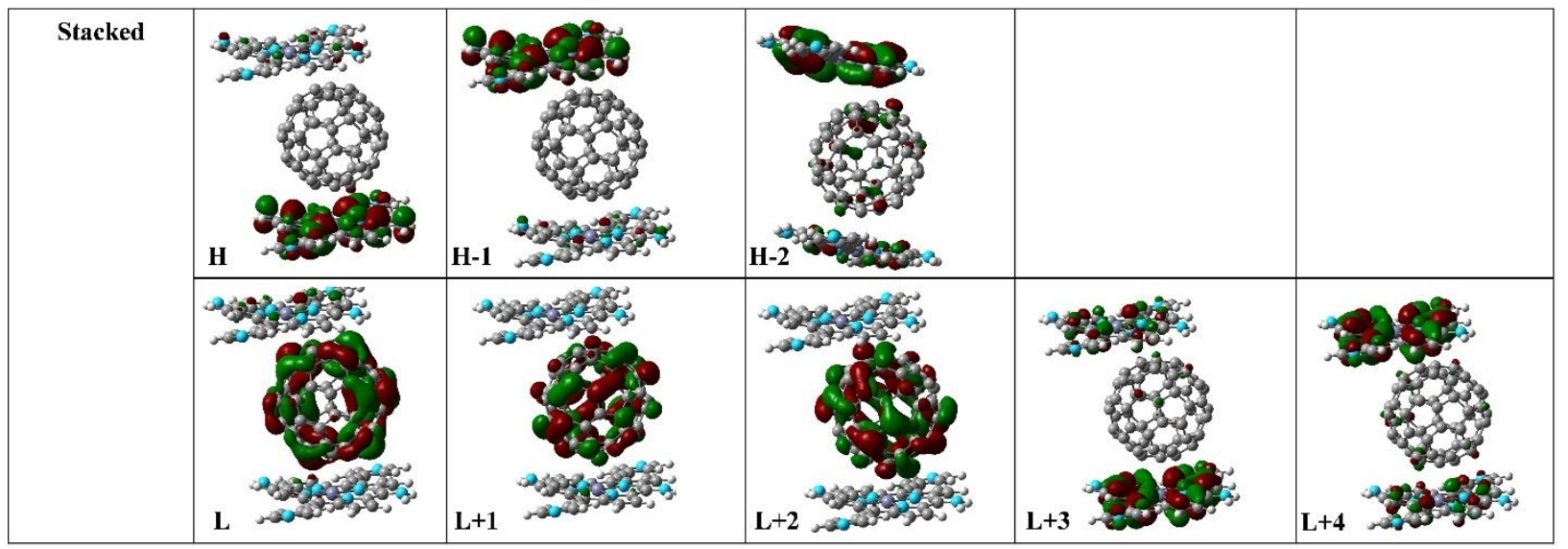

Figure S23. Frontier molecular orbitals for Type-II interface of $\mathrm{NH}_{2}$ DA-MOF in stacked fragments.

TableS10. Excited State, excitation energies (eV), wavelength (nm), oscillator strengths (f) and important orbitals involved in transition for Type-II interfaces of $\mathrm{NH}_{2}$ DA-MOF in their stacked fragments.

\begin{tabular}{|c|c|c|c|c|c|}
\hline $\begin{array}{c}\text { Systems } \\
\text { with } \\
\mathrm{C}_{60}\end{array}$ & $\begin{array}{c}\text { Excited State } \\
\text { (Ex. St.) }\end{array}$ & $\begin{array}{c}\text { Excitation energies } \\
\qquad\left(\mathrm{E}_{0-\mathrm{n}}, \mathrm{eV}\right)\end{array}$ & $\begin{array}{c}\text { Wavelength } \\
\text { (nm) }\end{array}$ & $\begin{array}{c}\text { Oscillator } \\
\text { strengths } \\
(f)\end{array}$ & $\begin{array}{c}\text { Important } \\
\text { Orbitals } \\
\text { involved }\end{array}$ \\
\hline \multirow{3}{*}{ Stacked } & 1 & 0.9997 & 1240.26 & 0.0008 & $\begin{array}{c}\mathrm{H}-1 \rightarrow \mathrm{L} \\
\mathrm{H} \rightarrow \mathrm{L} \\
\mathrm{H} \rightarrow \mathrm{L}+1\end{array}$ \\
\hline & 2 & 1.0277 & 1206.40 & 0.0180 & $\begin{array}{c}\mathrm{H}-1 \rightarrow \mathrm{L} \\
\mathrm{H}-1 \rightarrow \mathrm{L}+1 \\
\mathrm{H} \rightarrow \mathrm{L} \\
\mathrm{H} \rightarrow \mathrm{L}+1\end{array}$ \\
\hline & 9 & 1.5932 & 778.19 & 0.1201 & $\begin{array}{r}\mathrm{H}-1 \rightarrow \mathrm{L}+4 \\
\mathrm{H} \rightarrow \mathrm{L}+3 \\
\mathrm{H} \rightarrow \mathrm{L}+4\end{array}$ \\
\hline
\end{tabular}




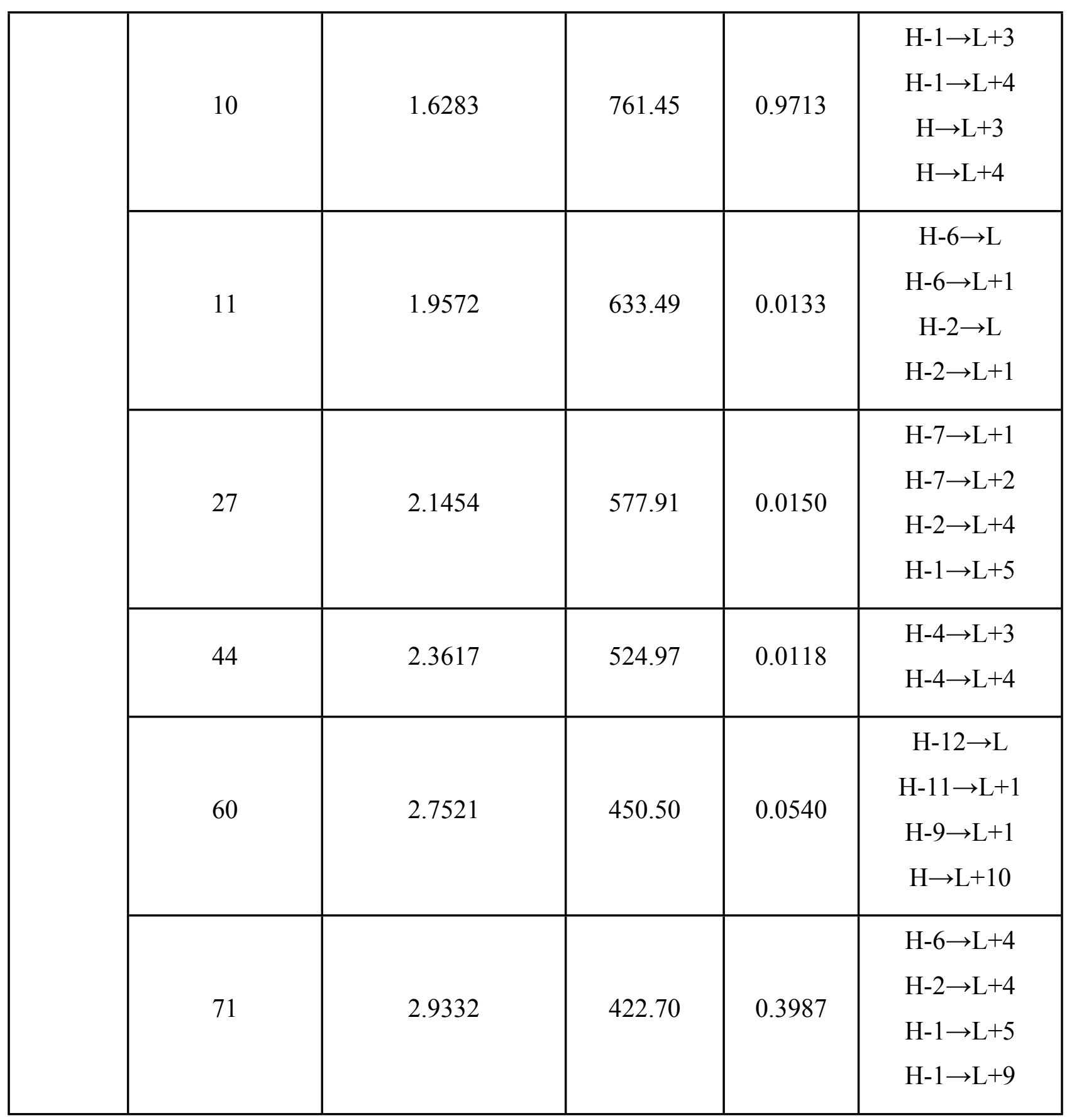


S2.10. Results for $\mathrm{C}_{60}$ incorporated $\mathrm{NH}_{2}$ DA-C60 in Type-III interface.

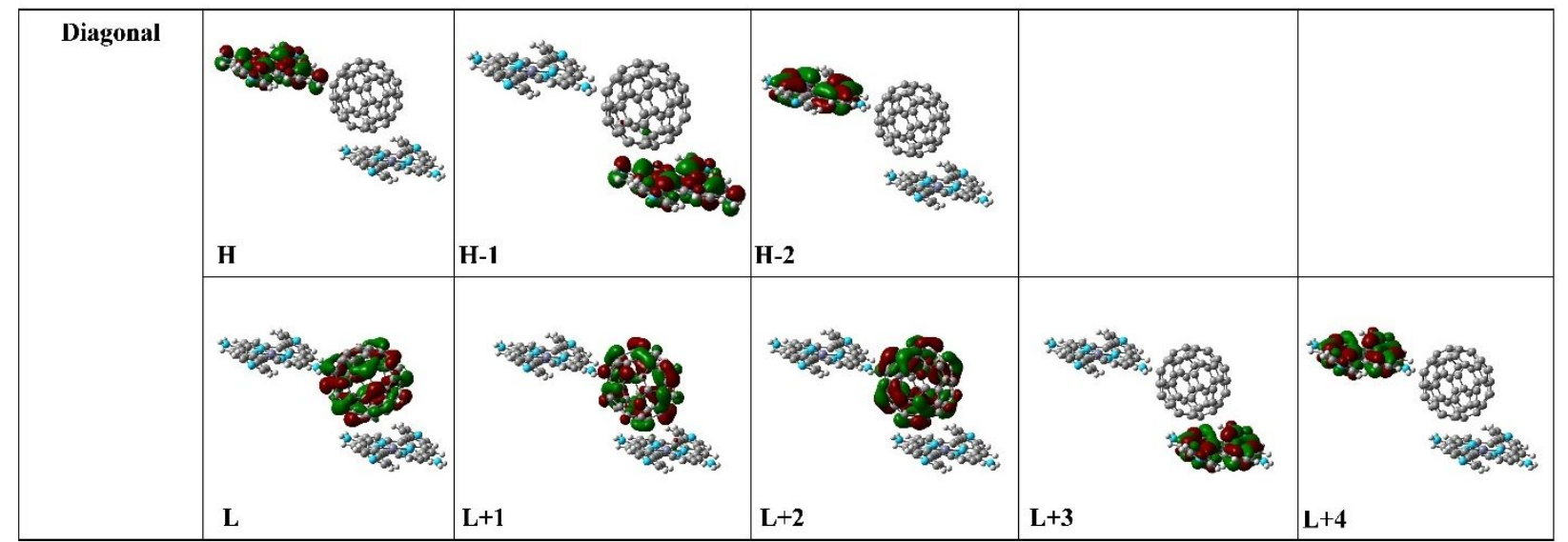

Figure S24. Frontier molecular orbitals for Type-III interface of $\mathrm{NH}_{2}$ DA-MOF in diagonal fragments.

TableS11. Excited State, excitation energies (eV), wavelength (nm), oscillator strengths (f) and important orbitals involved in transition for Type-III interfaces of $\mathrm{NH}_{2} \mathrm{DA}-\mathrm{MOF}$ in their diagonal fragments.

\begin{tabular}{|c|c|c|c|c|c|}
\hline $\begin{array}{c}\text { Systems } \\
\text { with } \mathrm{C}_{60}\end{array}$ & $\begin{array}{c}\text { Excited State } \\
\text { (Ex. St.) }\end{array}$ & $\begin{array}{c}\text { Excitation energies } \\
\left(\mathrm{E}_{0-\mathrm{n}}, \mathrm{eV}\right)\end{array}$ & $\begin{array}{c}\text { Wavelength } \\
(\mathrm{nm})\end{array}$ & $\begin{array}{c}\text { Oscillator } \\
\text { strengths } \\
(f)\end{array}$ & $\begin{array}{c}\text { Important } \\
\text { Orbitals } \\
\text { involved }\end{array}$ \\
\hline \multirow{2}{*}{ Diagonal } & 1 & 0.4497 & 2757.13 & 0.0033 & $\begin{array}{c}\mathrm{H} \rightarrow \mathrm{L} \\
\mathrm{H} \rightarrow \mathrm{L}+1\end{array}$ \\
\cline { 2 - 6 } & 2 & $0 . \mathrm{L}+56$ & 2548.15 & 0.0214 & $\begin{array}{c}\mathrm{H} \rightarrow \mathrm{L} \\
\mathrm{H} \rightarrow \mathrm{L}+1 \\
\mathrm{H} \rightarrow \mathrm{L}+2\end{array}$ \\
\cline { 2 - 6 } & 3 & 0.5091 & 2435.15 & 0.0236 & $\begin{array}{c}\mathrm{H} \rightarrow \mathrm{L} \\
\mathrm{H} \rightarrow \mathrm{L}+1 \\
\mathrm{H} \rightarrow \mathrm{L}+2\end{array}$ \\
\hline
\end{tabular}




\begin{tabular}{|c|c|c|c|c|}
\hline 6 & 0.7867 & 1575.94 & 0.0440 & $\begin{array}{c}\mathrm{H}-1 \rightarrow \mathrm{L} \\
\mathrm{H}-1 \rightarrow \mathrm{L}+1 \\
\mathrm{H}-1 \rightarrow \mathrm{L}+2\end{array}$ \\
\hline 13 & 1.6114 & 769.42 & 0.2801 & $\begin{array}{c}\mathrm{H}-1 \rightarrow \mathrm{L}+3 \\
\mathrm{H} \rightarrow \mathrm{L}+4 \\
\mathrm{H} \rightarrow \mathrm{L}+5\end{array}$ \\
\hline 14 & 1.6216 & 764.57 & 0.6765 & $\begin{array}{c}\mathrm{H}-2 \rightarrow \mathrm{L}+9 \\
\mathrm{H}-1 \rightarrow \mathrm{L}+3 \\
\mathrm{H} \rightarrow \mathrm{L}+4 \\
\mathrm{H} \rightarrow \mathrm{L}+5\end{array}$ \\
\hline 16 & 1.6879 & 734.56 & 0.0208 & $\mathrm{H} \rightarrow \mathrm{L}+7$ \\
\hline 23 & 1.9600 & 632.56 & 0.0139 & $\begin{array}{l}\mathrm{H}-1 \rightarrow \mathrm{L}+5 \\
\mathrm{H}-1 \rightarrow \mathrm{L}+6 \\
\mathrm{H}-1 \rightarrow \mathrm{L}+7\end{array}$ \\
\hline 62 & 2.6918 & 460.59 & 0.0827 & $\begin{array}{c}\mathrm{H}-5 \rightarrow \mathrm{L}+3 \\
\mathrm{H}-3 \rightarrow \mathrm{L}+3 \\
\mathrm{H}-1 \rightarrow \mathrm{L}+10 \\
\mathrm{H} \rightarrow \mathrm{L}+11\end{array}$ \\
\hline 92 & 2.8861 & 429.60 & 0.2460 & $\begin{array}{l}\mathrm{H}-4 \rightarrow \mathrm{L}+4 \\
\mathrm{H}-3 \rightarrow \mathrm{L}+3 \\
\mathrm{H}-2 \rightarrow \mathrm{L}+4\end{array}$ \\
\hline
\end{tabular}


S2.11. Results for $\mathrm{C}_{60}$ incorporated $\mathrm{NH}_{2} \mathrm{~F}-\mathrm{C} 60$ in Type-A interface.

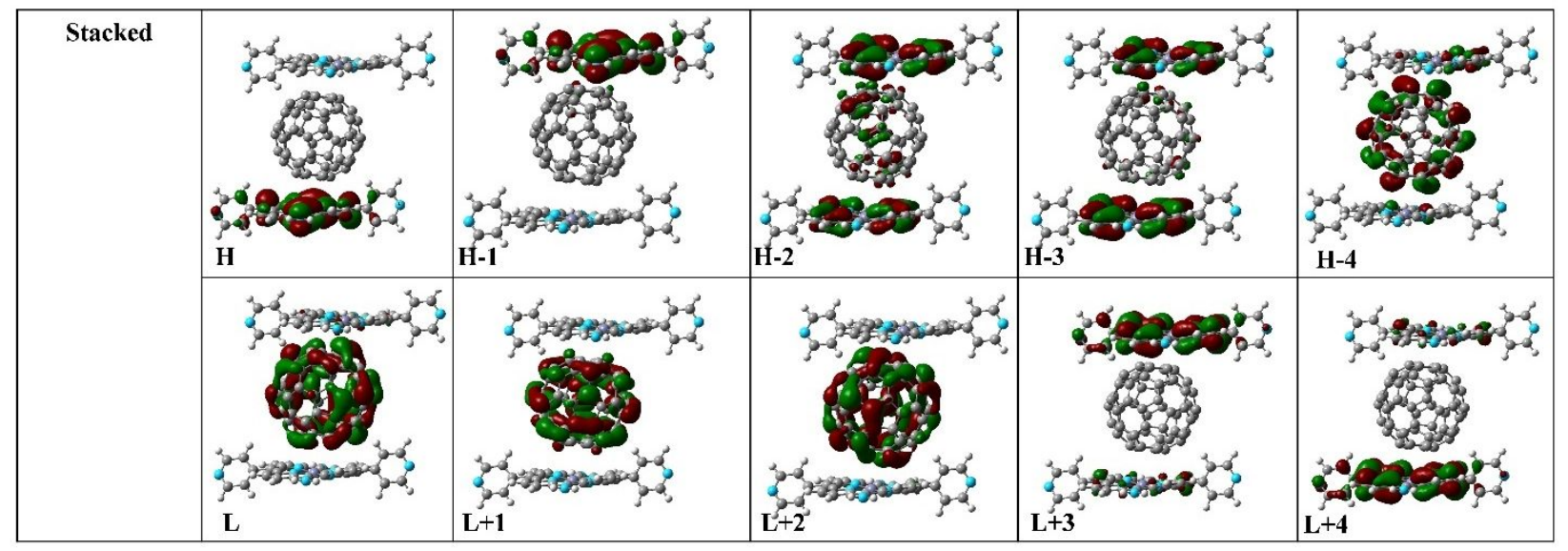

Figure S25. Frontier molecular orbitals for Type-A interface of $\mathrm{NH}_{2}$ F-MOF in stacked fragments.

TableS12. Excited State, excitation energies (eV), wavelength (nm), oscillator strengths (f) and important orbitals involved in transition for Type-A interfaces of $\mathrm{NH}_{2} \mathrm{~F}-\mathrm{MOF}$ in their stacked fragments.

\begin{tabular}{|c|c|c|c|c|c|}
\hline $\begin{array}{c}\text { Systems } \\
\text { with } \\
\mathrm{C}_{60}\end{array}$ & $\begin{array}{c}\text { Excited State } \\
\text { (Ex. St.) }\end{array}$ & $\begin{array}{c}\text { Excitation energies } \\
\left(\mathrm{E}_{0-\mathrm{n}}, \mathrm{eV}\right)\end{array}$ & $\begin{array}{c}\text { Wavelength } \\
(\mathrm{nm})\end{array}$ & $\begin{array}{c}\text { Oscillator } \\
\text { strengths } \\
(f)\end{array}$ & $\begin{array}{c}\text { Important } \\
\text { Orbitals } \\
\text { involved }\end{array}$ \\
\hline \multirow{3}{*}{ Stacked } & 1 & 0.8814 & 1406.68 & 0.0036 & $\begin{array}{c}\mathrm{H} \rightarrow \mathrm{L} \\
\mathrm{H} \rightarrow \mathrm{L}+1\end{array}$ \\
\cline { 2 - 6 } & 2 & 0.9256 & 1339.54 & 0.0087 & $\begin{array}{c}\mathrm{H}-1 \rightarrow \mathrm{L}-1 \rightarrow \mathrm{L}+1 \\
\mathrm{H} \rightarrow \mathrm{L}+1\end{array}$ \\
\cline { 2 - 6 } & 4 & 0.9343 & 1326.98 & 0.0346 & $\begin{array}{c}\mathrm{H}-1 \rightarrow \mathrm{L}+1 \\
\mathrm{H} \rightarrow \mathrm{L} \\
\mathrm{H} \rightarrow \mathrm{L}+1\end{array}$ \\
\hline
\end{tabular}




\begin{tabular}{|c|c|c|c|c|}
\hline & & & & $\mathrm{H} \rightarrow \mathrm{L}+2$ \\
\hline 6 & 0.9839 & 1260.19 & 0.0522 & $\begin{array}{c}\mathrm{H}-1 \rightarrow \mathrm{L} \\
\mathrm{H}-1 \rightarrow \mathrm{L}+1 \\
\mathrm{H}-1 \rightarrow \mathrm{L}+2 \\
\mathrm{H} \rightarrow \mathrm{L}+1\end{array}$ \\
\hline 10 & 1.8182 & 681.91 & 0.1874 & $\begin{array}{l}\mathrm{H}-2 \rightarrow \mathrm{L} \\
\mathrm{H} \rightarrow \mathrm{L}+3 \\
\mathrm{H} \rightarrow \mathrm{L}+4\end{array}$ \\
\hline 21 & 2.0472 & 605.64 & 0.0166 & $\begin{array}{c}\mathrm{H}-8 \rightarrow \mathrm{L} \\
\mathrm{H}-5 \rightarrow \mathrm{L} \\
\mathrm{H}-5 \rightarrow \mathrm{L}+1 \\
\mathrm{H}-4 \rightarrow \mathrm{L}+1 \\
\mathrm{H}-4 \rightarrow \mathrm{L}+2 \\
\mathrm{H}-1 \rightarrow \mathrm{L}+5\end{array}$ \\
\hline 37 & 2.2608 & 548.41 & 0.0115 & $\begin{array}{c}\mathrm{H}-8 \rightarrow \mathrm{L} \\
\mathrm{H}-8 \rightarrow \mathrm{L}+1 \\
\mathrm{H}-7 \rightarrow \mathrm{L}+1 \\
\mathrm{H}-7 \rightarrow \mathrm{L}+2 \\
\mathrm{H}-6 \rightarrow \mathrm{L} \\
\mathrm{H} \rightarrow \mathrm{L}+9\end{array}$ \\
\hline 50 & 2.7243 & H-1.10 & 0.0143 & $\begin{array}{c}\mathrm{H}-10 \rightarrow \mathrm{L} \\
\mathrm{H}-5 \rightarrow \mathrm{L}+3 \\
\mathrm{H}-4 \rightarrow \mathrm{L}+3 \\
\mathrm{H}-3 \rightarrow \mathrm{L}+3 \\
\mathrm{H}-2 \rightarrow \mathrm{L}+3\end{array}$ \\
\hline 65 & 3.0315 & 408.98 & 0.0307 & $\begin{array}{l}\mathrm{H}-11 \rightarrow \mathrm{L}+1 \\
\mathrm{H}-11 \rightarrow \mathrm{L}+2 \\
\mathrm{H}-3 \rightarrow \mathrm{L}+5\end{array}$ \\
\hline
\end{tabular}




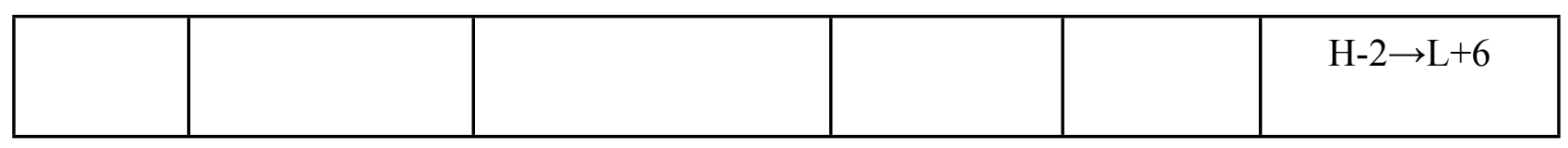

S2.12. Results for $\mathrm{C}_{60}$ incorporated $\mathrm{NH}_{2}$ F-C60 in Type-B interface.

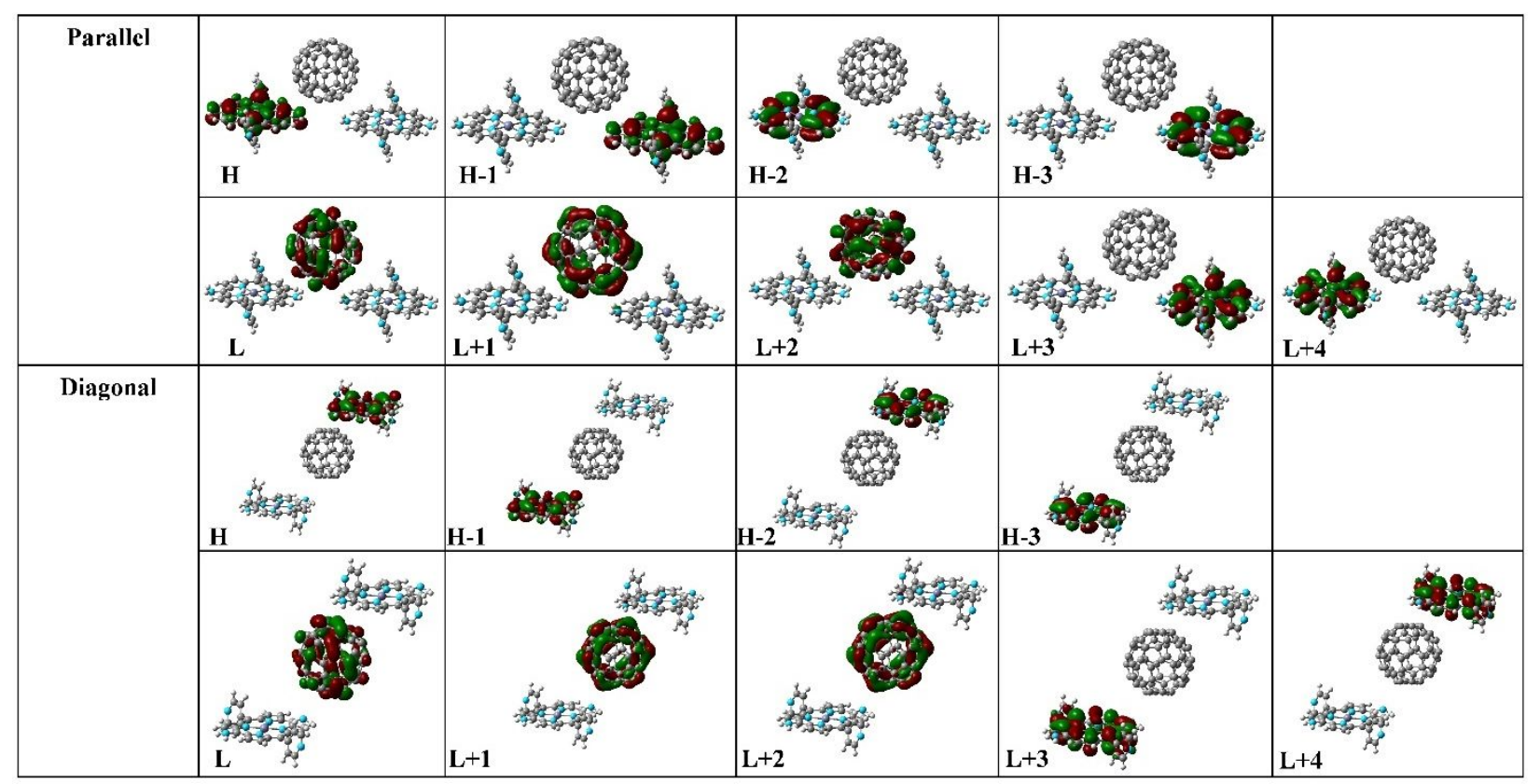

Figure S26. Frontier molecular orbitals for Type-B interface of $\mathrm{NH}_{2}$ F-MOF in parallel and diagonal fragments.

TableS13. Excited State, excitation energies (eV), wavelength (nm), oscillator strengths (f) and important orbitals involved in transition for Type-A interfaces of $\mathrm{NH}_{2}$ F-MOF in their parallel and diagonal fragments.

\begin{tabular}{|c|c|c|c|c|c|}
\hline $\begin{array}{c}\text { Systems } \\
\text { with } \mathrm{C}_{60}\end{array}$ & $\begin{array}{c}\text { Excited State } \\
(\text { Ex. St. })\end{array}$ & $\begin{array}{c}\text { Excitation energies } \\
\left(\mathrm{E}_{0-\mathrm{n}}, \mathrm{eV}\right)\end{array}$ & $\begin{array}{c}\text { Wavelength } \\
(\mathrm{nm})\end{array}$ & $\begin{array}{c}\text { Oscillator } \\
\text { strengths } \\
(f)\end{array}$ & $\begin{array}{c}\text { Important } \\
\text { Orbitals } \\
\text { involved }\end{array}$ \\
\hline Parallel & 1 & 0.5161 & 2402.37 & 0.0010 & $\mathrm{H} \rightarrow \mathrm{L}$ \\
\hline
\end{tabular}




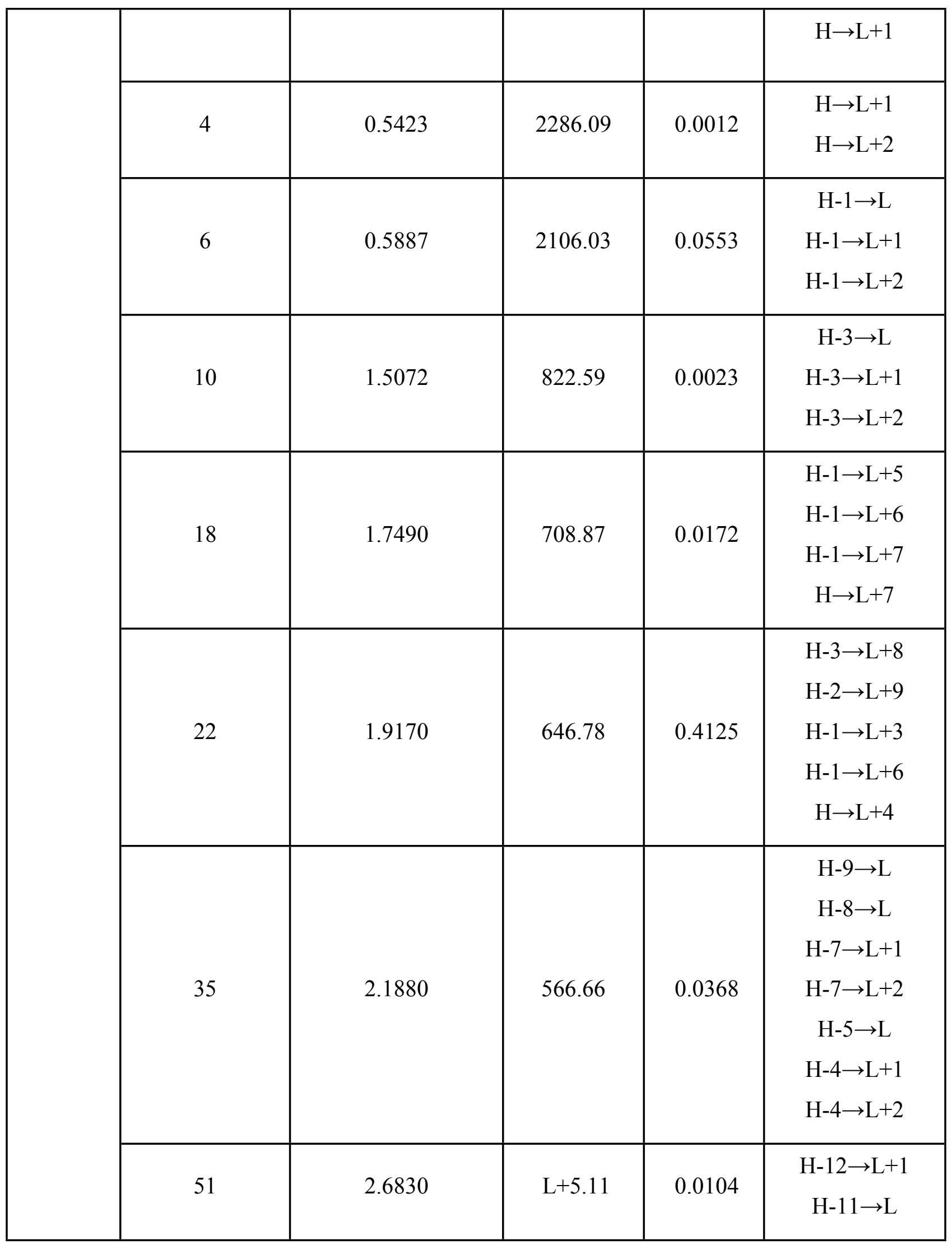




\begin{tabular}{|c|c|c|c|c|c|}
\hline & & & & & $\begin{array}{l}\mathrm{H}-11 \rightarrow \mathrm{L}+1 \\
\mathrm{H}-11 \rightarrow \mathrm{L}+2\end{array}$ \\
\hline & 99 & 2.9835 & 415.57 & 0.0101 & $\begin{array}{l}\mathrm{H}-1 \rightarrow \mathrm{L}+13 \\
\mathrm{H}-1 \rightarrow \mathrm{L}+14\end{array}$ \\
\hline \multirow{8}{*}{ Diagonal } & 1 & 0.5318 & 2331.48 & 0.0002 & $\begin{array}{c}\mathrm{H} \rightarrow \mathrm{L} \\
\mathrm{H} \rightarrow \mathrm{L}+1\end{array}$ \\
\hline & 2 & 0.5565 & 2227.85 & 0.0013 & $\begin{array}{c}\mathrm{H} \rightarrow \mathrm{L} \\
\mathrm{H} \rightarrow \mathrm{L}+1 \\
\mathrm{H} \rightarrow \mathrm{L}+2\end{array}$ \\
\hline & 4 & 0.5951 & 2083.48 & 0.0114 & $\begin{array}{c}\mathrm{H}-1 \rightarrow \mathrm{L}+1 \\
\mathrm{H} \rightarrow \mathrm{L}+1 \\
\mathrm{H} \rightarrow \mathrm{L}+2\end{array}$ \\
\hline & 5 & 0.6064 & 2044.56 & 0.0412 & $\begin{array}{c}\mathrm{H}-1 \rightarrow \mathrm{L}+1 \\
\mathrm{H}-1 \rightarrow \mathrm{L}+2 \\
\mathrm{H} \rightarrow \mathrm{L}+1 \\
\mathrm{H} \rightarrow \mathrm{L}+2\end{array}$ \\
\hline & 7 & 1.5121 & 819.97 & 0.0037 & $\begin{array}{c}\mathrm{H}-2 \rightarrow \mathrm{L} \\
\mathrm{H}-2 \rightarrow \mathrm{L}+2\end{array}$ \\
\hline & 16 & 1.7618 & 703.73 & 0.0131 & $\begin{array}{l}\mathrm{H} \rightarrow \mathrm{L}+5 \\
\mathrm{H} \rightarrow \mathrm{L}+6 \\
\mathrm{H} \rightarrow \mathrm{L}+4\end{array}$ \\
\hline & 22 & 1.9186 & 646.23 & 0.3305 & $\begin{array}{l}\mathrm{H}-3 \rightarrow \mathrm{L}+8 \\
\mathrm{H}-1 \rightarrow \mathrm{L}+3\end{array}$ \\
\hline & 34 & 2.2006 & 563.41 & 0.0396 & $\begin{array}{c}\mathrm{H}-5 \rightarrow \mathrm{L}+1 \\
\mathrm{H}-4 \rightarrow \mathrm{L}+2 \\
\mathrm{H} \rightarrow \mathrm{L}+9\end{array}$ \\
\hline
\end{tabular}




\begin{tabular}{|c|c|c|c|c|c|}
\hline & & & & & $\mathrm{H}-12 \rightarrow \mathrm{L}$ \\
$\mathrm{H}-2 \rightarrow \mathrm{L}+4$ \\
\end{tabular}


Section S3. Anisotropic hopping pathways for hole and electron migration.

The Marcus equation that (Equation S1) is extensively used to calculate the hopping rate $(k)$ between adjacent molecules:

$k=\frac{2 \pi V^{2}}{h}\left(\frac{\pi}{\lambda k_{b} T}\right)^{\frac{1}{2}} \exp \left(-\frac{\left(\lambda+\Delta G^{0}\right)^{2}}{4 \lambda k_{b} T}\right) \quad \ldots \ldots \ldots . .$. Equation $\mathrm{S} 1$

Here, $V$ is the charge transfer integral (or electronic coupling) between the initial and final states, $\lambda$ is the reorganization energy, $h$ is Plank's constant, $k_{b}$ is the Boltzmann constant and $\mathrm{T}$ is the absolute temperature and $\Delta G^{0}$ is the total Gibbs free energy change for the electron transfer reaction. 


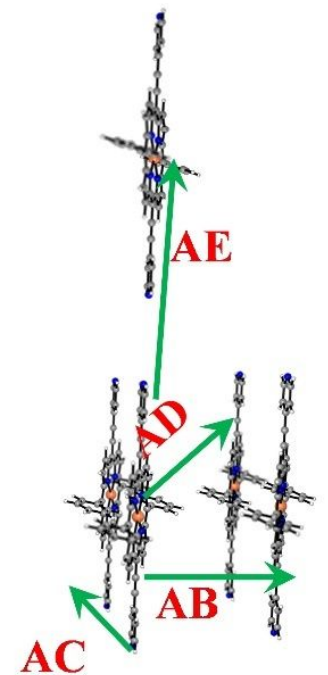

(a)

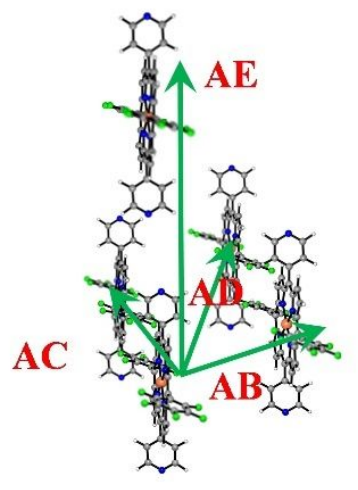

(d)

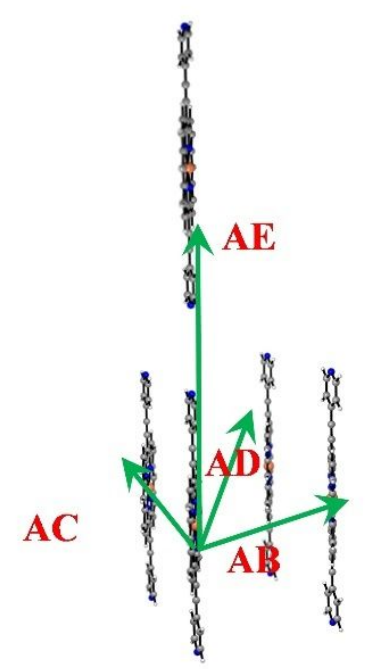

(b)

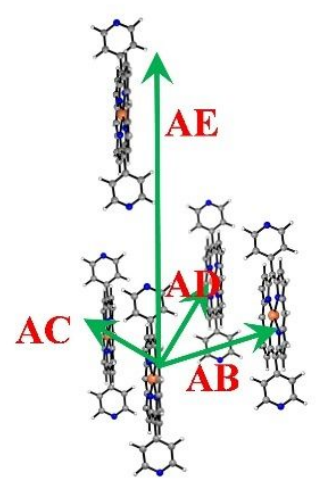

(e)

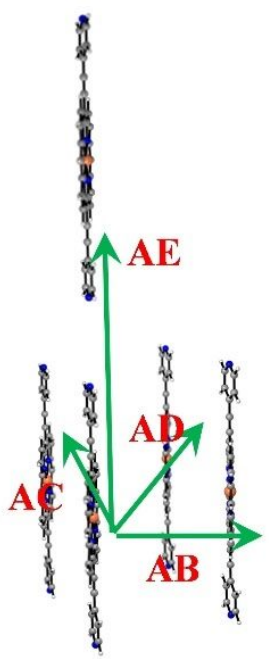

(c)

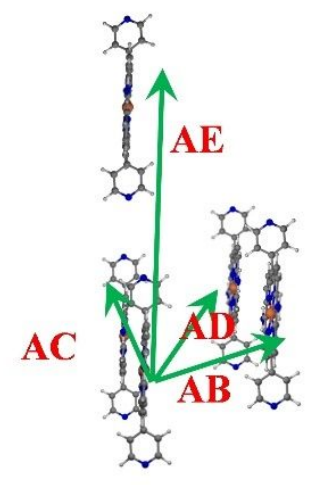

(f)

Figure S27. Hopping pathways between adjacent porphyrin units in (a) DA-MOF (b) H_DAMOF (c) $\mathrm{NH}_{2}$ DA-MOF (d) F-MOF (e) H_F-MOF and (f) $\mathrm{NH}_{2}$ F-MOF. Center to center distances between adjacent porphyrins are shown in Table S14. 
TableS14. Computed charge transfers integral values along different pathways for DA-MOF, H_DA-MOF, NH ${ }_{2}$ DA-MOF, F-MOF, H_F-MOF and $\mathrm{NH}_{2}$ F-MOF. Center to center distances between adjacent porphyrins are shown in parenthesis.

\begin{tabular}{|c|c|c|c|c|c|c|c|c|}
\hline & $V_{h}\left(\mathrm{~cm}^{-1}\right)$ & $V_{\mathrm{e}}\left(\mathrm{cm}^{-1}\right)$ & $V_{h}\left(\mathrm{~cm}^{-1}\right)$ & $V_{\mathrm{e}}\left(\mathrm{cm}^{-1}\right)$ & $V_{h}\left(\mathrm{~cm}^{-1}\right)$ & $V_{e}\left(\mathrm{~cm}^{-1}\right)$ & $V_{h}\left(\mathbf{c m}^{-1}\right)$ & $V_{e}\left(\mathrm{~cm}^{-1}\right)$ \\
\hline DA- & \multicolumn{2}{|c|}{$\mathrm{AB}(11.44)$} & \multicolumn{2}{|c|}{$\mathrm{AC}(15.70)$} & \multicolumn{2}{|c|}{$\mathrm{AD}(19.43)$} & \multicolumn{2}{|c|}{$\mathrm{AE}(27.323)$} \\
\hline MOF & 0.080655 & 0 & 0 & 0.080655 & 2.09704 & 0.56459 & 0.08066 & 0.080655 \\
\hline \multirow{2}{*}{$\begin{array}{c}\text { H_DA- } \\
\text { MOF }\end{array}$} & \multicolumn{2}{|c|}{$\mathrm{AB}(11.48)$} & \multicolumn{2}{|c|}{$\mathrm{AC}(15.72)$} & \multicolumn{2}{|c|}{$\mathrm{AD}(19.48)$} & \multicolumn{2}{|c|}{$\operatorname{AE}(27.38)$} \\
\hline & 0.08066 & 0 & 0 & 0 & 0.080655 & 0.08066 & 0.08066 & 0.080655 \\
\hline \multirow{2}{*}{$\begin{array}{c}\mathrm{NH}_{2} \mathrm{DA} \\
\mathrm{MOF}\end{array}$} & \multicolumn{2}{|c|}{$\mathrm{AB}(11.48)$} & \multicolumn{2}{|c|}{$\mathrm{AC}(15.72)$} & \multicolumn{2}{|c|}{$\mathrm{AD}(19.48)$} & \multicolumn{2}{|c|}{$\operatorname{AE}(27.38)$} \\
\hline & 0.16131 & 0 & 0 & 0 & 0.241966 & 0.080655 & 0.08066 & 0.080655 \\
\hline \multirow{2}{*}{ F-MOF } & \multicolumn{2}{|c|}{$\mathrm{AB}(11.59)$} & \multicolumn{2}{|c|}{$\mathrm{AC}(15.46)$} & \multicolumn{2}{|c|}{$\mathrm{AD}(19.27)$} & \multicolumn{2}{|c|}{$\mathrm{AE}(22.06)$} \\
\hline & 0 & 0.080655 & 0 & 0 & 0.241966 & 7.66226 & 0 & 0 \\
\hline \multirow{2}{*}{$\begin{array}{l}\text { H_F- } \\
\text { MOF }\end{array}$} & \multicolumn{2}{|c|}{$\mathrm{AB}(11.63)$} & \multicolumn{2}{|c|}{$\mathrm{AC}(15.48)$} & \multicolumn{2}{|c|}{$\mathrm{AD}(19.32)$} & \multicolumn{2}{|c|}{$\mathrm{AE}(22.09)$} \\
\hline & 0.080655 & 0.080655 & 0 & 0 & 0 & 0 & 0 & 0.080655 \\
\hline \multirow{2}{*}{$\begin{array}{c}\mathrm{NH}_{2} \mathrm{~F}- \\
\mathrm{MOF}\end{array}$} & \multicolumn{2}{|c|}{$\mathrm{AB}(11.63)$} & \multicolumn{2}{|c|}{$\mathrm{AC}(15.48)$} & \multicolumn{2}{|c|}{$\mathrm{AD}(19.32)$} & \multicolumn{2}{|c|}{$\mathrm{AE}(22.09)$} \\
\hline & \begin{tabular}{|l}
0.080655 \\
\end{tabular} & 0.080655 & 0 & 0 & 0.161311 & 0.161311 & 0.08066 & 0.08066 \\
\hline
\end{tabular}



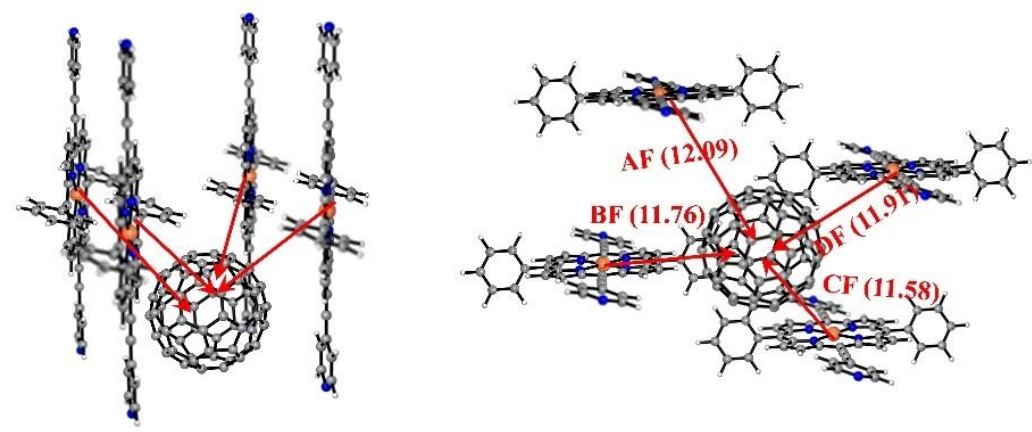

(a)
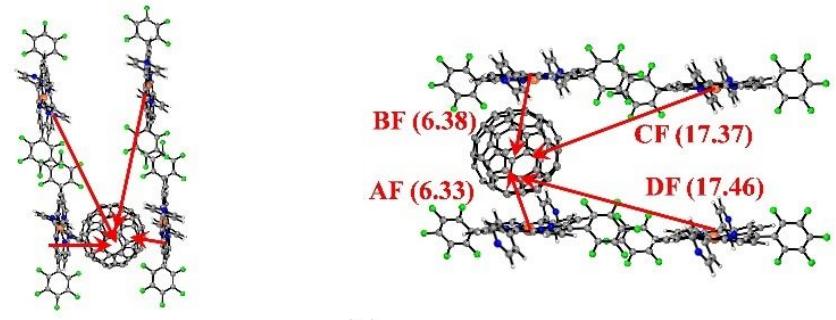

(b)

Figure S28. Hopping pathways between adjacent porphyrin units and $\mathrm{C}_{60}$ in $\mathrm{C}_{60}$ incorporated (a) DA-MOF and (b) F-MOF. Center to center distances between adjacent porphyrins and $\mathrm{C}_{60}$ are shown in $\AA$. 

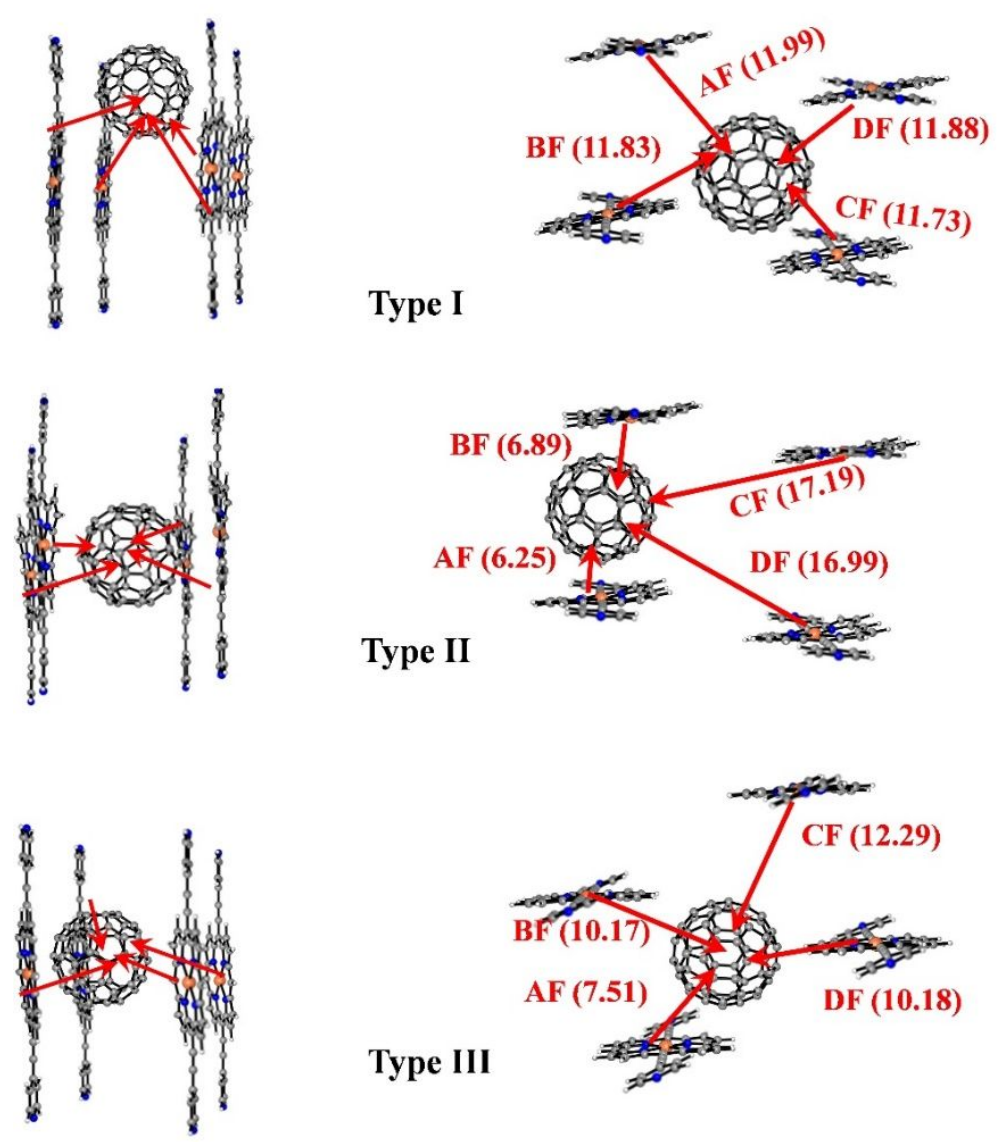

Figure S29. Hopping pathways between adjacent porphyrin units and $\mathrm{C}_{60}$ in Type-I, Type-II and Type-III interfaces for H_DA-MOF. Center to center distances between adjacent porphyrins and $\mathrm{C}_{60}$ are shown in $\AA$. 

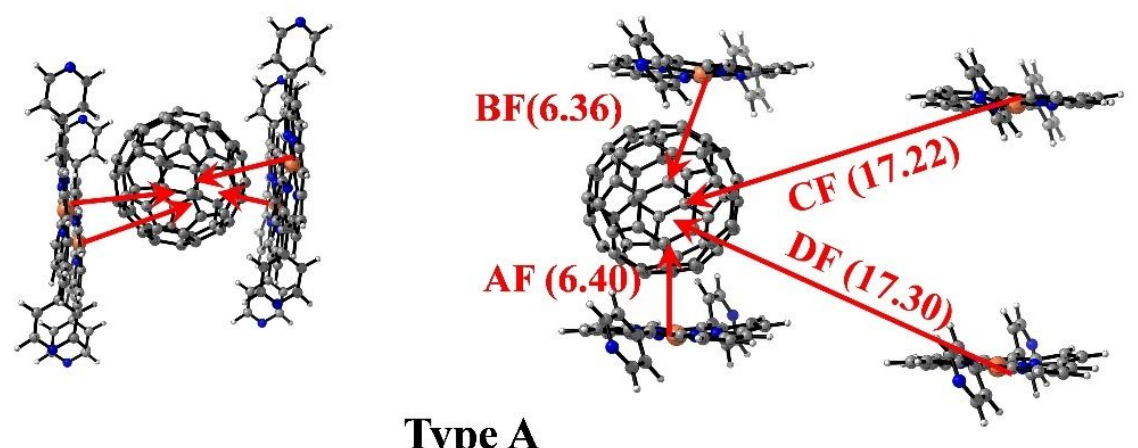

Type A
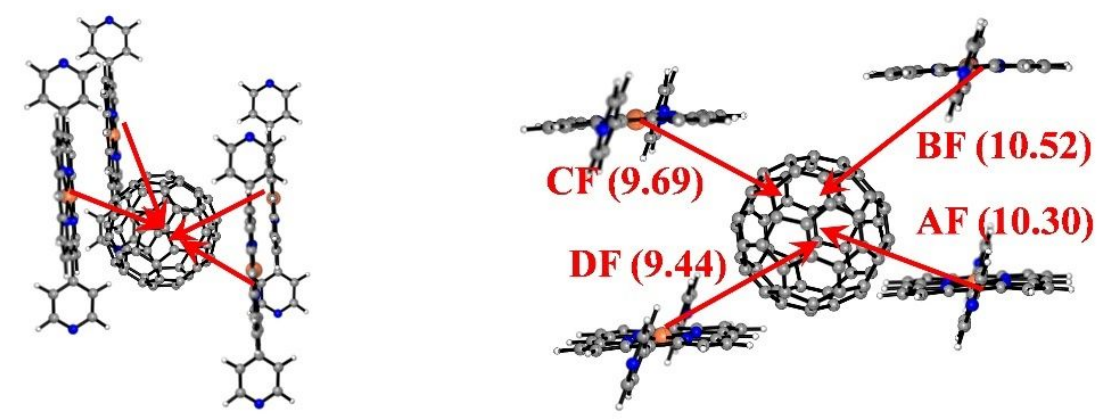

Type B

Figure S30. Hopping pathways between adjacent porphyrin units and $\mathrm{C}_{60}$ in Type-A and Type-B interfaces for H_F-MOF. Center to center distances between adjacent porphyrins and $\mathrm{C}_{60}$ are shown in $\AA$. 

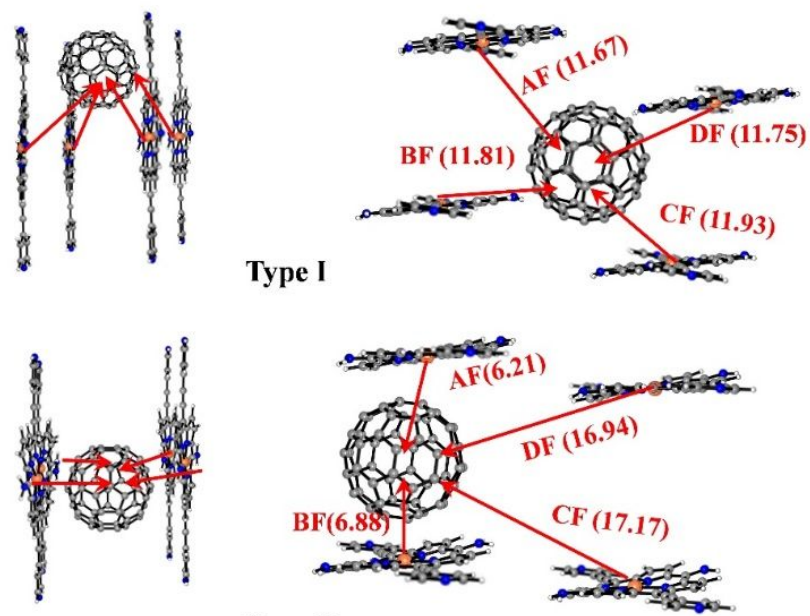

Type II
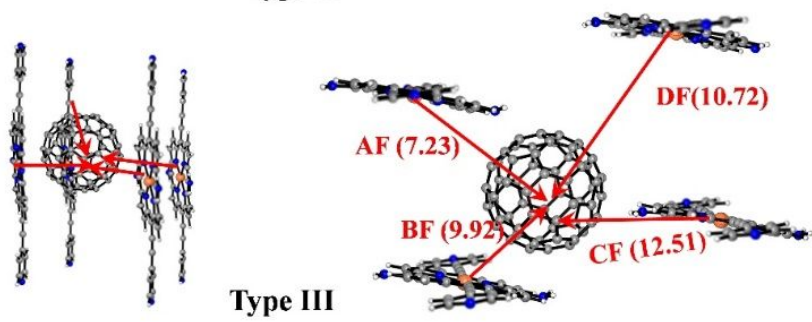

Figure S31. Hopping pathways between adjacent porphyrin units and $\mathrm{C}_{60}$ in Type-I, Type-II and Type-III interfaces for $\mathrm{NH}_{2}$ DA-MOF. Center to center distances between adjacent porphyrins and $\mathrm{C}_{60}$ are shown in $\AA$. 

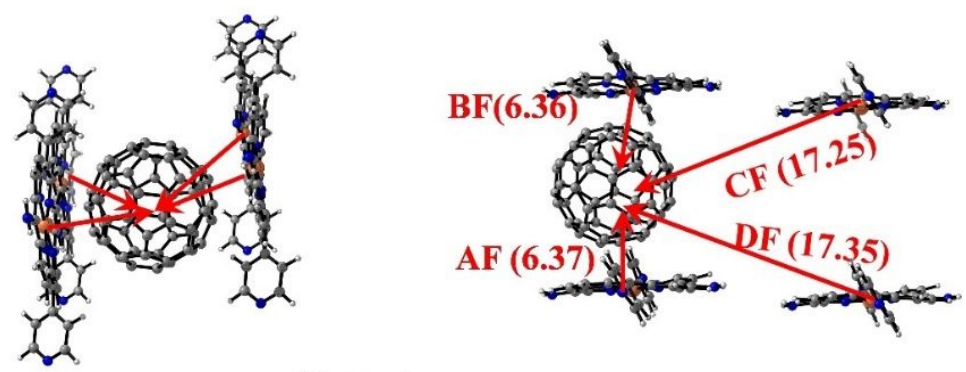

Type A

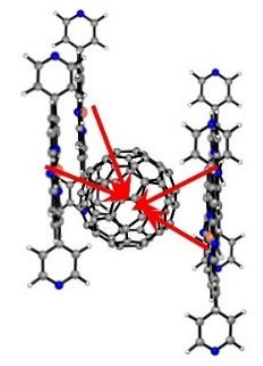

Type B

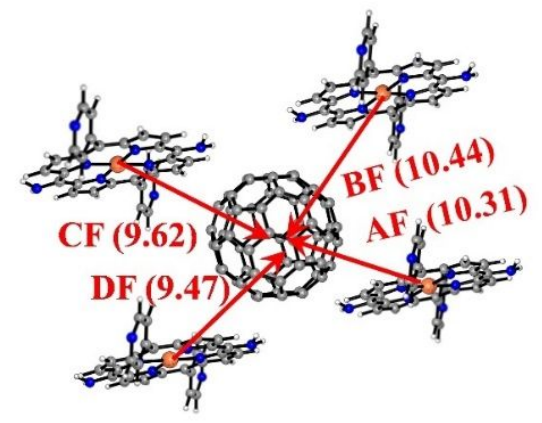

Figure S32. Hopping pathways between adjacent porphyrin units and $\mathrm{C}_{60}$ in Type-A and Type-B interfaces for $\mathrm{NH}_{2}$ F-MOF. Center to center distances between adjacent porphyrins and $\mathrm{C}_{60}$ are shown in $\AA$.

TableS15. Highest obtained charge transfers integral values (pathway is given in parenthesis) for DA-MOF, H_DA-MOF, NH ${ }_{2}$ DA-MOF, F-MOF, H_F-MOF, $\mathrm{NH}_{2}$ F-MOF and their $\mathrm{C}_{60}$ incorporated analogues. The order of increase after $\mathrm{C}_{60}$ installation is also reported.

\begin{tabular}{|c|c|c|c|c|}
\hline & \multicolumn{2}{|c|}{ Transfer Integral (V) in $\mathrm{cm}^{-1}$} & \multicolumn{2}{c|}{ Order of increase } \\
\hline & hole & electron & hole & electron \\
\hline DA-MOF & $2.1(\mathrm{AD})$ & $0.6(\mathrm{AD})$ & -- & - \\
\hline DA-MOF+C60 & $82.1(\mathrm{BF})$ & $9.5(\mathrm{DF})$ & 39.2 & 16.8 \\
\hline
\end{tabular}




\begin{tabular}{|c|c|c|c|c|}
\hline H_DA-MOF & $0.1(\mathrm{AB})$ & 0.1 (AE) & -- & -- \\
\hline $\begin{array}{c}\text { H_DA-MOF+C60 } \\
\text { Type-I }\end{array}$ & 19.9 (DF) & $5.6(\mathrm{DF})$ & 247 & 70 \\
\hline $\begin{array}{c}\text { H_DA-MOF+C60 } \\
\text { Type-II }\end{array}$ & $774.3(\mathrm{AF})$ & $695.2(\mathrm{BF})$ & 9600 & 8619 \\
\hline $\begin{array}{c}\text { H_DA-MOF+C60 } \\
\text { Type-III }\end{array}$ & $687.5(\mathrm{AF})$ & $392.8(\mathrm{AF})$ & 8524 & 4870 \\
\hline NH2_DA-MOF & $0.2(\mathrm{AD})$ & $0.1(\mathrm{AE})$ & -- & -- \\
\hline $\begin{array}{c}\text { NH2_DA-MOF+C60 } \\
\text { Type-I }\end{array}$ & $11.5(\mathrm{AF})$ & 13.8 (DF) & 47.7 & 171 \\
\hline $\begin{array}{c}\mathrm{NH} 2 \_\mathrm{DA}-\mathrm{MOF}+\mathrm{C} 60 \\
\text { Type-II }\end{array}$ & 992.9 (AF) & $669.4(\mathrm{BF})$ & 4103.7 & 8299 \\
\hline $\begin{array}{c}\mathrm{NH} 2 \_\mathrm{DA}-\mathrm{MOF}+\mathrm{C} 60 \\
\text { Type-III }\end{array}$ & $610.7(\mathrm{AF})$ & 627.1 (DF) & 2524 & 2835 \\
\hline F-MOF & $0.2(\mathrm{AD})$ & $7.7(\mathrm{AD})$ & -- & -- \\
\hline $\mathrm{F}-\mathrm{MOF}+\mathrm{C} 60$ & $383.6(\mathrm{BF})$ & $534.7(\mathrm{BF})$ & 1585.3 & 69.8 \\
\hline H_F-MOF & $0.1(\mathrm{AB})$ & $0.1(\mathrm{AB} / \mathrm{AE})$ & -- & -- \\
\hline $\begin{array}{c}\text { H_F-MOF+C60 } \\
\text { Type-A }\end{array}$ & 297.9 (BF) & $591.3(\mathrm{BF})$ & 1847 & -3665.5 \\
\hline
\end{tabular}




\begin{tabular}{|c|c|c|c|c|}
\hline $\begin{array}{c}\text { H_F-MOF+C60 } \\
\text { Type-B }\end{array}$ & $194.1(\mathrm{CF})$ & $212.5(\mathrm{DF})$ & 2406 & 2635 \\
\hline NH2_F-MOF & $0.2(\mathrm{AD})$ & $0.2(\mathrm{AD})$ & -- & -- \\
\hline $\begin{array}{c}\text { NH2_F-MOF+C60 } \\
\text { Type-A }\end{array}$ & $427.6(\mathrm{BF})$ & $406.5(\mathrm{BF})$ & 2650.5 & 2520 \\
\hline $\begin{array}{c}\text { NH2_F-MOF+C60 } \\
\text { Type-B }\end{array}$ & $410.2(\mathrm{DF})$ & $190.2(\mathrm{DF})$ & 2543 & 1179 \\
\hline
\end{tabular}

\section{Section S4. Full references of Gaussian 16 and ADF.}

(i) Complete Reference for Gaussian 16: Gaussian 16, Revision B.01, Frisch, M. J.; Trucks, G. W.; Schlegel, H. B.; Scuseria, G. E.; Robb, M. A.; Cheeseman, J. R.; Scalmani, G.; Barone, V.; Petersson, G. A.; Nakatsuji, H.; Li, X.; Caricato, M.; Marenich, A. V.; Bloino, J.; Janesko, B. G.; Gomperts, R.; Mennucci, B.; Hratchian, H. P.; Ortiz, J. V.; Izmaylov, A. F.; Sonnenberg, J. L.; Williams-Young, D.; Ding, F.; Lipparini, F.; Egidi, F.; Goings, J.; Peng, B.; Petrone, A.; Henderson, T.; Ranasinghe, D.; Zakrzewski, V. G.; Gao, J.; Rega, N.; Zheng, G.; Liang, W.; Hada, M.; Ehara, M.; Toyota, K.; Fukuda, R.; Hasegawa, J.; Ishida, M.; Nakajima, T.; Honda, Y.; Kitao, O.; Nakai, H.; Vreven, T.; Throssell, K.; Montgomery, J. A., Jr.; Peralta, J. E.; Ogliaro, F.; Bearpark, M. J.; Heyd, J. J.; Brothers, E. N.; Kudin, K. N.; Staroverov, V. N.; Keith, T. A.; Kobayashi, R.; Normand, J.; Raghavachari, K.; Rendell, A. P.; Burant, J. C.; Iyengar, S. S.; Tomasi, J.; Cossi, M.; Millam, J. M.; Klene, M.; Adamo, C.; Cammi, R.; Ochterski, J. W.; Martin, R. L.; Morokuma, K.; Farkas, O.; Foresman, J. B.; Fox, D. J. Gaussian, Inc., Wallingford CT, 2016. 
(ii) Complete Reference for Amsterdam Density Functional software (ADF): ADF2019, SCM, Theoretical Chemistry, Vrije Universiteit, Amsterdam, The Netherlands, E.J. Baerends, T. Ziegler, A.J. Atkins, J. Autschbach, O. Baseggio, D. Bashford, A. Bérces, F.M. Bickelhaupt, C. Bo, P.M. Boerrigter, L. Cavallo, C. Daul, D.P. Chong, D.V. Chulhai, L. Deng, R.M. Dickson, J.M. Dieterich, D.E. Ellis, M. van Faassen, L. Fan, T.H. Fischer, C. Fonseca Guerra, M. Franchini, A. Ghysels, A. Giammona, S.J.A. van Gisbergen, A. Goez, A.W. Götz, J.A. Groeneveld, O.V. Gritsenko, M. Grüning, S. Gusarov, F.E. Harris, P. van den Hoek, Z. Hu, C.R. Jacob, H. Jacobsen, L. Jensen, L. Joubert, J.W. Kaminski, G. van Kessel, C. König, F. Kootstra, A. Kovalenko, M.V. Krykunov, E. van Lenthe, D.A. McCormack, A. Michalak, M. Mitoraj, S.M. Morton, J. Neugebauer, V.P. Nicu, L. Noodleman, V.P. Osinga, S. Patchkovskii, M. Pavanello, C.A. Peeples, P.H.T. Philipsen, D. Post, C.C. Pye, H. Ramanantoanina, P. Ramos, W. Ravenek, J.I. Rodríguez, P. Ros, R. Rüger, P.R.T. Schipper, D. Schlüns, H. van Schoot, G. Schreckenbach, J.S. Seldenthuis, M. Seth, J.G. Snijders, M. Solà, M. Stener, M. Swart, D. Swerhone, V. Tognetti, G. te Velde, P. Vernooijs, L. Versluis, L. Visscher, O. Visser, F. Wang, T.A. Wesolowski, E.M. van Wezenbeek, G. Wiesenekker, S.K. Wolff, T.K. Woo, A.L. Yakovlev. 